\title{
Étude de l'action de la force de Coriolis sur la propagation des marées en fonction de l'étendue de la zone marine considérée
}

\author{
Investigation of the effect of the Coriolis \\ force on the propagation of tides as a function \\ of the size of the region under consideration
}

\author{
PAR J. VALEMBOIS ET R. BONNEFILLE
}

ingtenieurs aU laboratoire National D'HYDraUliQue

\begin{abstract}
Un modèle réduit tournant, schématisant une mer de forme triangulaire à fond incliné, a été construit au Laboratoire National d'Hydraulique, pour étudier expérimentalement l'influence de la force de Coriolis sur la marée en fonction des dimensions de l'étendue marine représentée par le modèle.

Dans le cas d'une mer $\dot{a}$ fond rugueux, de profondeur variant entre 0 et quelques centaines de mètres, l'influence de la force de Coriolis se traduit par une déformation de l'onde-marée encore sensible pour les mers de plus de $300 \mathrm{~km}$ de côté, mais négligeable pour les bassins maritimes inférieurs à $150 \mathrm{~km}$. On a, par contre, observé une modification profonde du champ de courants en ce qui concerne le sens de rotation du vecteur vitesse dans l'espace, ce phénomène étant indépendant de l'étendue de la zone marine considérée.

La même étude, avec fond lisse, conduit à un régime d'onde stationnaire qui masque l'influence de la force de Coriolis sur les marnages. Par contre, le champ de courants est alors très différent de celui obtenu avec un fond très rugueux. L'étude de la marée pour diverses valeurs du frottemenl fail apparaîle une variation continue de la physionomie du champ de courants, se traduisant principalement par l'inversion du sens de giration du vecteur vitesse.
\end{abstract}

\begin{abstract}
A rotating model which diagrammatically simulates a triangular sea with a sloping bed, was constructed at the National Hydraulic Laboratory for the purpose of investigating the effect of the Coriolis force as a function of the size of the region simulated on the model.

When the seabed is rough and its depth varies between zero and a feul hundred metres, the effect of the Coriolis force is to deform the tide wave. This is still perceptible when one shore of the sea is $300 \mathrm{~km}$ long, but is negligible when this length is less than $150 \mathrm{~km}$. However, a fundamental modification of the current field was noticed in connection with the direction of rotation of the velocity vector in space, this effect being independent of the size of the region under consideration.

Similar experiments with a smooth seabed give rise to a standing wave which hides the effect of the Coriolis force. Here the current field differs very greatly from that encountered when the seabed is very rough. Consideration of the tide with different coefficients of friction shows that the variation of the current field is continuous and lhat il has as its main effect the reversal of the direction of rotation of the velocity vector.
\end{abstract}

\section{I. - INTRODUCTION}

L'étude de l'effet de la force de Coriolis due à la rotation terrestre sur le phénomène de la propagation de la marée retrouve un regain d'actualité avec les études préliminaires du projet de l'usine marémotrice des îles Chausey. En effet, l'éventualité d'effectuer les études hydrauliques du projet (remous de l'usine sur la marée, courants pendant les phases de construction de l'usine..) sur des modèles tournants de grande dimension, amène d'une part 
une grande complication technique et d'autre part une dépense d'étude qui peut paraître excessive eu égard à la précision demandée dans les résultats.

Il n'est pas physiquement évident que la force de Coriolis n'ait qu'une part négligeable, même du point de vue de l'ingénieur, en ce qui concerne l'interprétation des résultats des modèles réduits, du fait de la grande période du phénomène et de l'importance de la zone marine intéressée par le projet. Cependant, compte tenu de l'importance du frottement sur les fonds de faible profondeur du golfe de Saint-Malo, de la complexité du dessin des côtes qui entraîne corrélativement une grande complexité du champ de courant, on est conduit à croire qu'un modèle non tournant est suffisant pour représenter le phénomène de la marée dans la zone intéressée et avec une précision jugée acceptable.
Une des contributions du Laboratoire National d'Hydraulique au sein de la Commission du Pool Coriolis chargée de se prononcer sur l'éventualité de faire tourner les modèles réduits hydrauliques nécessaires à ces études, a été d'examiner sur un petit modèle tournant l'influence relative de la force de Coriolis sur la marée en fonction de l'aire marine représentée par le modèle. Cette étude a été effectuée dans un bassin tournant de forme géométrique simple pour une condition aux limites donnée, soit avec un fond rugueux, soit avec un fond lisse. Le programme d'essais a pour but d'étudier l'effet de la force de Coriolis dans les zones maritimes dont les dimensions vont de lordre du millier de kilomètres et où la force de Coriolis est prépondérante, jusqu'aux bassins de quelques dizaines de kilomètres de longueur où la fore de Coriolis est sûrement néğligeable.

\section{II. - CONSIDÉRATIONS GÉNERALES ET SIMILITUDE}

L'idée directrice de l'étude est de déterminer la réduction de l'influence relative de la force de Coriolis au fur et à mesure que diminue la surface de l'aire marine reproduite sur le modèle réduit. En effet, pour que la force de Coriolis puisse arriver à modifier profondément l'écoulement d'un filet fluide, il faut que l'intégrale de son action le long de ce filet fluide s'effectue tout au long d'une distance suffisante. On com prend ainsi que la force de Coriolis arrive à modifier la direction de propagation des grands courants marins et la physionomie de la marée, en Manche centrale par exemple. Par contre, si l'on considère un petit domaine maritime, le long de la frontière duquel les conditions naturelles de marées sont bien reproduites, on concoit mal que l'adjonction de la force de Coriolis aux forces hydrodynamiques classiques puisse modifier profondément le phénomène hydraulique.

Dans cet ordre d'idées, le bassin d'essai choisi a la forme d'une pyramide à section rectangulaire, dont une face latérale horizontale est représentée par la surface libre du fluide, la face opposée correspondant au fond du modèle incliné à partir du sommet, les deux autres faces étant les còtés verticaux du bassin. La base rectangulaire de la pyramide est matérialisée par la pelle du générateur de marée. Vu en plan, le bassin, c'est-à-dire la face supérieure de la pyramide, a la forme d'un triangle rectangle isocèle dont l'hypoténuse est la trace de la pelle du générateur de marée. Ce bassin peut ainsi, pour certaines échelles, schématiser le golfe de Saint-Malo ou une partie de celui-ci.

Si on transpose un phénomène hydraulique observé dans ce bassin, en utilisant une série d'échelles en plan et en conservant un rapport constant entre les échelles en plan et en hauteur, on obtient le phénomène qui se produirait dans une série de bassins géométriquement semblables, mais de dimensions différentes. Inversement, si on veut étudier un phénomène hydraulique périodique, de période donnée et constante, dans une série de bassins de mème forme, mais de dimensions différentes, on peut utiliser le modèle réduit unique de même forme géométrique, à condition de conserver un rapport constant $1 / \mathrm{D}$ entre les échelles de similitude $\lambda$ en plan et p. en hauteur. L'échelle des temps sur le modèle réduit est alors :

$$
\tau=\frac{\lambda}{\mu \cdot 2}=\left(\frac{\lambda}{\mu}\right)^{2} \lambda^{1 / 2}=D^{1 / 2} \lambda^{3 / 4}
$$

elle dépend de l'échelle en plan et donc elle varie d'une transposition hydraulique à l'autre.

Pratiquement nous étudions la marée semidiurne de période type 12 h $25 \mathrm{mn}=44700 \mathrm{~s}$, dans un volume fluide placé à la surface de la terre. La surface de cette zone marine est suffisamment faible pour négliger en première approximation la courbure de la terre et la variation locale de l'intensité de la composante verticale de la rotation terrestre. La région étudiée est supposée centrée à la latitude $49^{\circ}$ nord. On choisit le rapport de distorsion $\mathrm{D}=u / \lambda=100$, d'où $\tau=\lambda^{1 \%} / 10$. La période de la marée du modèle est alors : T $(s)=4470 \lambda^{1 / 2}$ et la période de la rotation du modèle :

$$
R(s)=\frac{86400}{\sin 49^{\circ}}=\frac{\lambda^{1 / 3}}{10}=11460 \lambda^{1 / 2}
$$




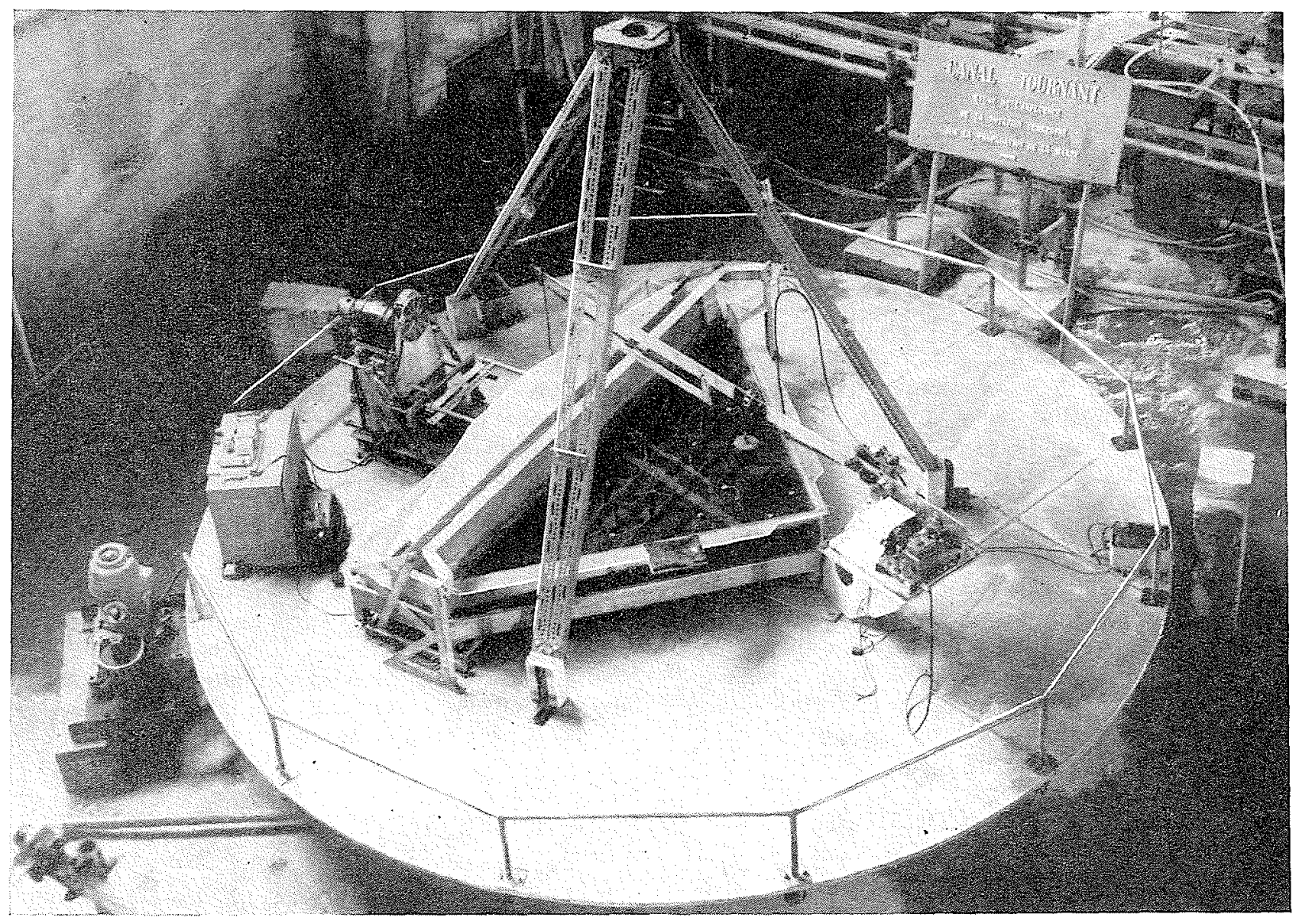

III

\section{DESCRIPTION DE L'INSTALLATION}

Sur la plateforme tournante de $4 \mathrm{~m}$ de diamètre est installé un bassin triangulaire rectangle et isocèle dont le côté de l'angle droit mesure $1,50 \mathrm{~m}$. Le fond de ce bassin a une pente uniforme et la profondeur varie ainsi de 0 au sommet de l'angle droit à $11 \mathrm{~cm}$ le long de l'hypoténuse. La pelle, de $2,1 \mathrm{~m}$ de long, du générateur de marée est disposée suivant l'hypoténuse du triangle. Elle est animée d'un mouvement de iranslation sinusoïdal et engendre l'onde-marée dans le bassin. L'amplitude de ce mouvement est réglable de 0 à $150 \mathrm{~mm}$. On peut faire varier la période de $5 \mathrm{~s}$ à $1 \mathrm{mn}$. La vitesse de rotation du bassin est réglable de 1 tour en $10 \mathrm{~s}$ à 1 tour en $1 \mathrm{mn}$. Cette rotation s'effectue dans le sens trigonométrique. Le centre de gravité du bassin est placé sur l'axe de rotation de la plateforme de façon à réduire l'effet de la force centrifuge.

Pour la mesure des marnages, une pointe vibrante asservie est montée sur un chariot se déplaçant sur un bras mobile autour d'un axe vertical situé au sommet de l'angle droit du triangle. Cette pointe est reliée par un système de fils et de poulies à un limnigraphe fixé sur le plateau tournant. Le déplacement du chariot est commandé électriquement de l'extérieur de la plateforme. On efrectue en chaque point un
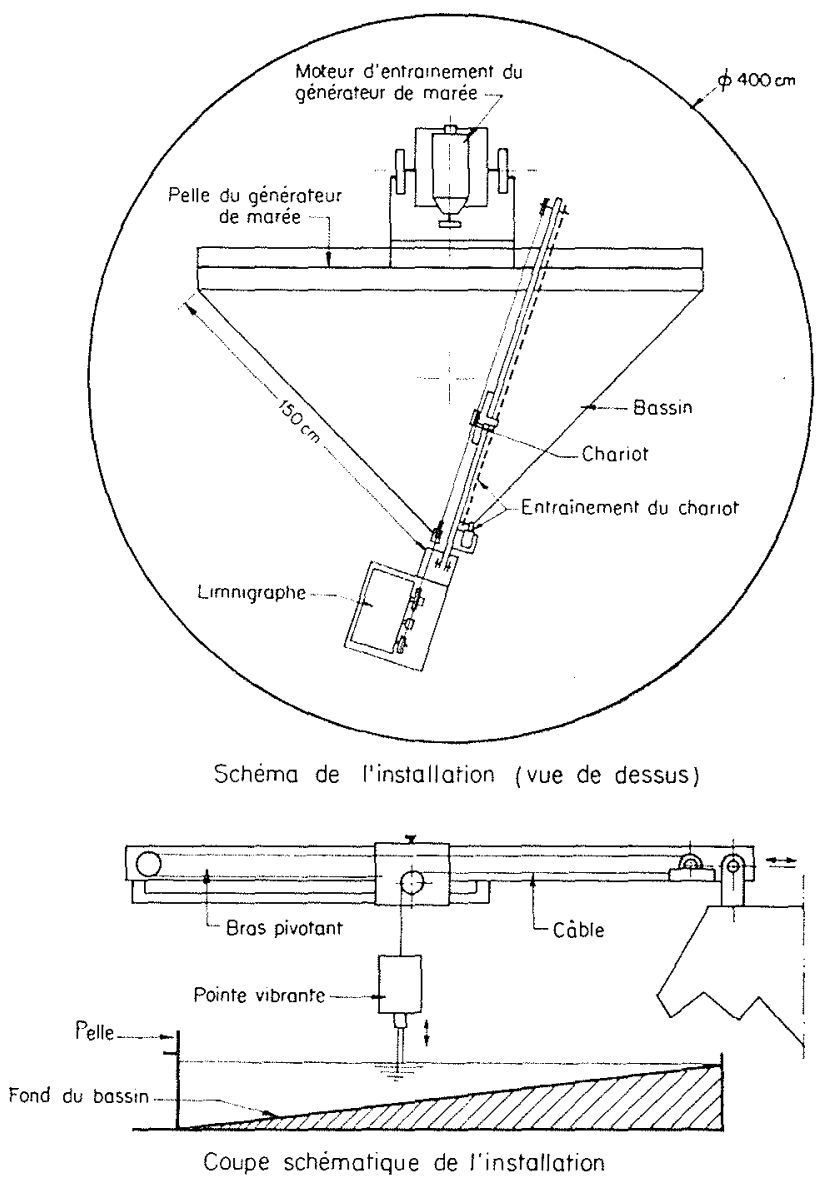

FIG. 1 
enregistrement continu du marnage pendant un nombre suffisant de périodes, de façon à obtenir une mesure moyenne des caractéristiques locales de l'onde-marée. Un top de synchronisation émis à un instant donné du cycle de la marée, permet de définir l'horaire de la progression de l'onde et ainsi de tracer les lignes cotidales.

Une caméra électrique fixée à $0,5 \mathrm{~m}$ au-dessus du bassin enregistre les trajectoires de flotteurs. Après étude, à l'analyseur de film, de la succession des positions des flotteurs, on en déduit la répartition des vitesses dans le triangle. Un système de tops de synchronisation et une horloge-marée placée dans le champ de la caméra permettent de relier ces vitesses à l'heure de la marée.

\section{IV. - CONDUITE DES ESSAIS}

Pour représenter les grands espaces marins sur le bassin de dimensions restreintes, et afin que ce dernier ait une profondeur suffisante, on est amené à choisir une distorsion importante égale à 100. L'échelle des temps étant donnée, il en résulte celle des longueurs et des profondeurs. Ainsi, si on fait varier la période du batteur, c'est-à-dire celle de la marée sur le modèle, les dimensions de ce dernier étant constantes, on représente un triangle nature plus ou moins grand. La période de rotation du bassin est lice à l'échelle des temps et par conséquent à la période de la marée modèle, et à la latitude du lieu représenté sur le modèle.

Pour chaque période de la marée modèle, on effectue trois essais, le premier avec rotation nulle, le second avec rotation $49^{\circ} \mathrm{N}$ correspon-

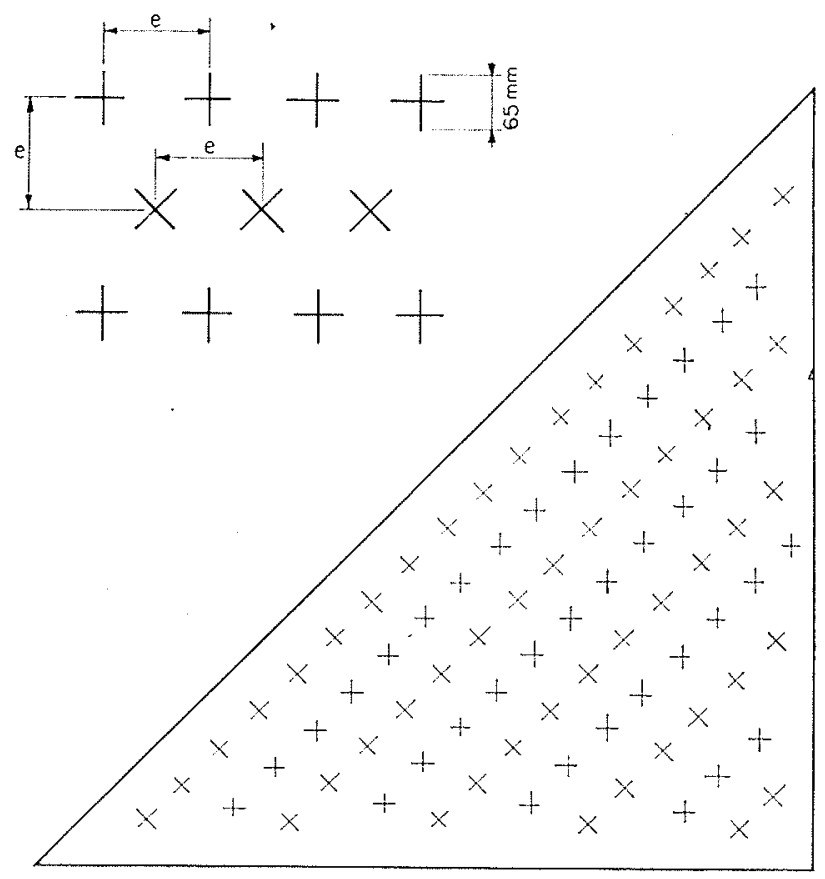

Fig. 2

Disposition schématique des éléments de rugosite. dant à la latitude du golfe de Saint-Malo, le troisieme en « rotation critique $»$, c'est-à-dire en considérant le triangle nature situé au voisinage du pôle; dans ce dernier cas, la période de rotation est double de celle du générateur de marée et correspond à l'étude de la marée dans un bassin placé aux latitudes critiques. Les essais sont effectués pour les périodes de marée modèle indiquées au tableau suivant, qui donne en outre, pour chaque période, les échelles et les grandeurs réelles représentées par le côté du bassin et sa profondeur.

La première série d'essais est effectuée avec in fond très rugueux, obtenu en disposant des

\begin{tabular}{|c|c|c|c|c|c|}
\hline \multirow[b]{2}{*}{$\begin{array}{l}\text { Echelle } \\
\text { en plan }\end{array}$} & \multirow[b]{2}{*}{$\begin{array}{c}\text { Echelle } \\
\text { en } \\
\text { hauteur }\end{array}$} & \multirow[b]{2}{*}{$\begin{array}{l}\text { Période } \\
\quad(s)\end{array}$} & \multirow[b]{2}{*}{$\begin{array}{c}\text { Rota- } \\
\text { tion } \\
49^{\circ} \mathrm{N} \\
(\mathrm{s})\end{array}$} & \multicolumn{2}{|c|}{$\begin{array}{l}\text { Cuve de } 1,5 \mathrm{~m} \\
\text { et } 11 \mathrm{~cm}\end{array}$} \\
\hline & & & & 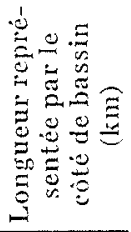 & 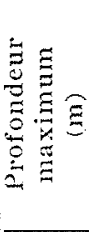 \\
\hline $1 / 600000$ & $1 / 6000$ & 5,77 & 14,8 & 900 & 660 \\
\hline $1 / 500000$ & $1 / 5000$ & 6,32 & 16,2 & 750 & 550 \\
\hline $1 / 400000$ & $1 / 4000$ & 7,07 & 18,1 & 600 & 440 \\
\hline $1 / 300000$ & $1 / 3000$ & 8,16 & 21,4 & 450 & 330 \\
\hline $1 / 200000$ & $1 / 2000$ & 10,00 & 25,6 & 300 & 220 \\
\hline $1 / 140000$ & $1 / 1400$ & 11,95 & 30,6 & 210 & 154 \\
\hline $1 / 100000$ & $1 / 1000$ & 14,15 & 36,4 & 150 & 110 \\
\hline $1 / 70000$ & $1 / 700$ & 16,9 & 43,3 & 105 & 77 \\
\hline $1 / 60000$ & $1 / 600$ & 18,2 & 46,8 & 90 & 66 \\
\hline $1 / 50000$ & $1 / 500$ & 20,0 & 51,2 & 75 & 55 \\
\hline $1 / 40000$ & 1/400 & 22,3 & $57,3$. & 60 & 44 \\
\hline
\end{tabular}


bandes de métal déployé verticales formant un carroyage d'environ 5 à $10 \mathrm{~cm}$ de côté. La deuxième série d'essais est effectuée en conservant le fond lisse. Enfin, une troisiène série d'essais est effectuéc avec un frottement variable permettant d'assurer la transition entre les deux séries d'essais précédentes. Ce frottement variable est obtenu en tapissant le fond du modèle par des croix en tôle. Celles-ci ont l'avantage d'être très efficaces du point de vue frottement, tout en étant non directionnelles si on les dispose en quinconce. Par modification de l'espacement entre chaque élément, on obtient la variation de la force de frottement (fig. 2).

\section{V. - ETUDE DES MARNAGES}

\section{$1^{\circ}$ Généralités.}

Les résultats relatifs à quelques mesures de marnage sont présentés sous la forme de réseaux de ligines d'égale amplitude de la marée et de lignes cotidales. Les figures 3 à 6 sont relatives aux essais avec frottement important et les figures 7 et 9 aux essais sans frottement. On a choisi l'échelle des amplitudes de marée égale à $1 / 1000^{\circ}$. Le fait que cette échelle soit différente

10 Lignes d'égale amplirude de la marée, en mètres - In-Lignes cotidales er avance de la maree, en heures
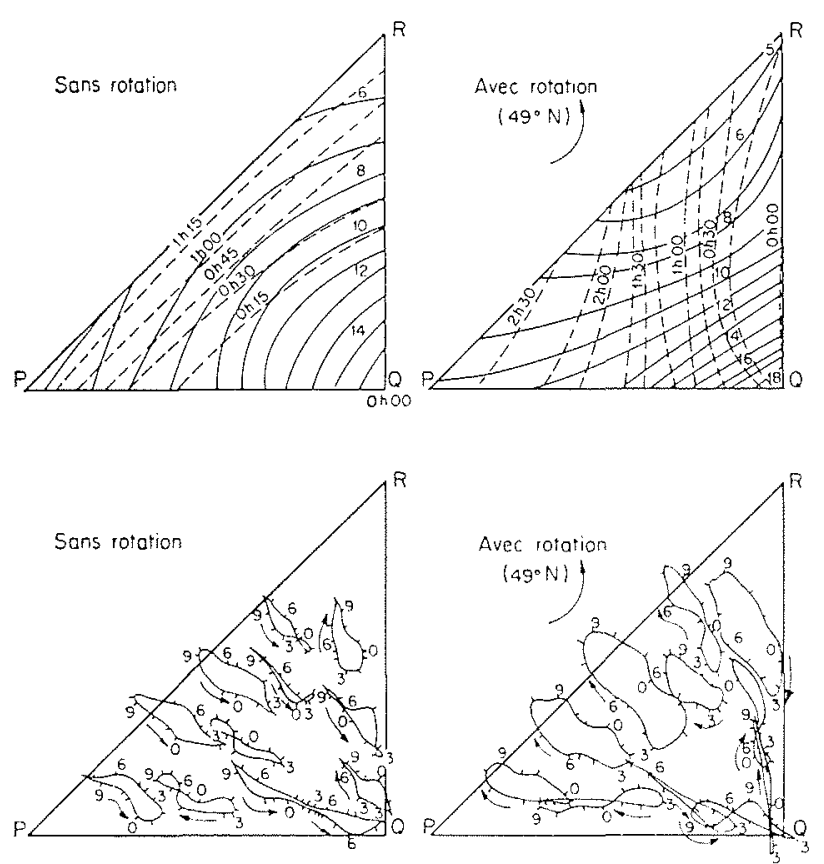

FIC. 3

Période de la maré : 5,77 s.

Amplitude du batteur : $44 \mathrm{~mm}$.

Période de la rotation de la plateforme: $14,8 \mathrm{~s}$.

Longueur du côté de l'angle droit : $900 \mathrm{lim}$.

Profondeur maximum : $660 \mathrm{~m}$.

Essais abec rugosite. de l'échelle en hauteur n'est gênant que dans la région voisine du sommet de l'angle droit, en raison de la faible profondeur dans cette zone. L'avantage de la valeur $1 / 1000^{\circ}$ est qu'elle conduit à des amplitudes de marée de l'ordre de $15 \mathrm{~m}$ dans la zone $\mathrm{k}$ sommet de l'angle droit, d'où un point de comparaison avee les marées du golfe de Saint-Malo.

On constate, Iorque le hassin est immobile, que les lignes d'égale amplitude de la marée et

\footnotetext{
$\stackrel{3}{-1}$ Position du vecteur vitesse dons te temps en heures

- Sens de rotation du vecteur vilesse

O. $5 \mathrm{~cm} / \mathrm{s}$ Echelle des viresses
}
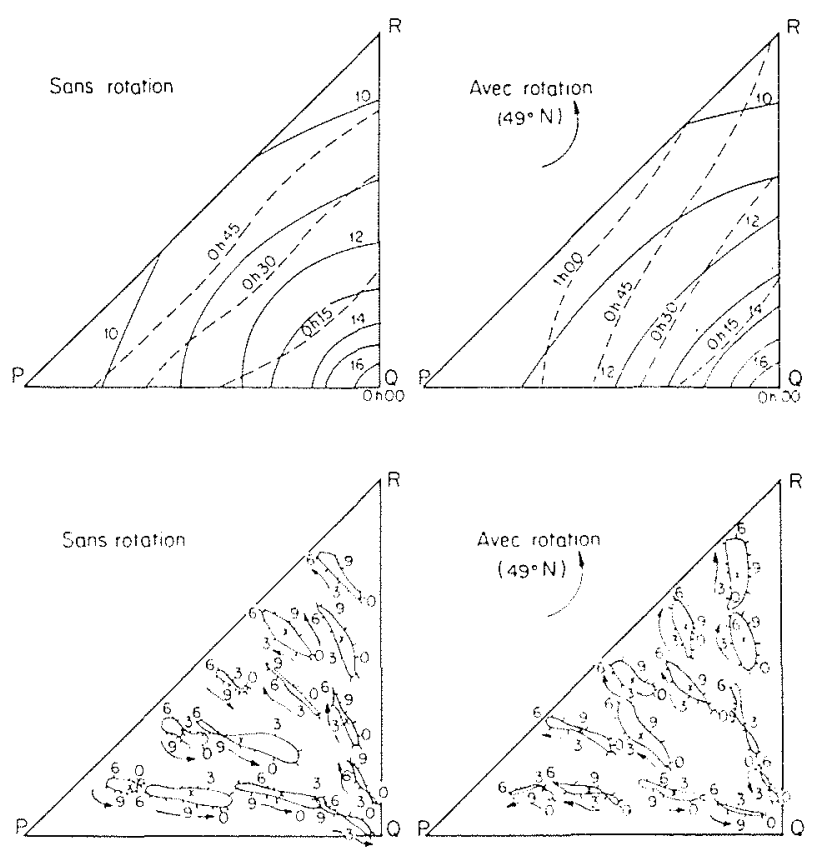

FIG. 4

Periode de la malée : $10 \mathrm{~s}$.

Amplitude du balteur : $80 \mathrm{~mm}$.

Période de la rotation de la plateforme : $25,6 \mathrm{~s}$

Longueur du coté de l'angle droit : $300 \mathrm{~km}$.

Profondeur maximum : $220 \mathrm{~m}$.

Essais avec rugosité. 

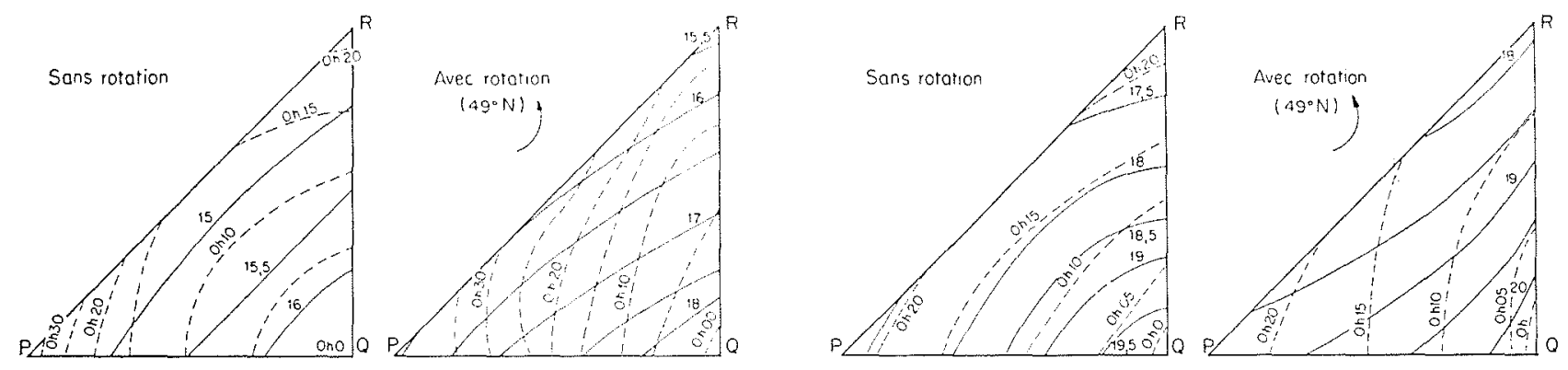

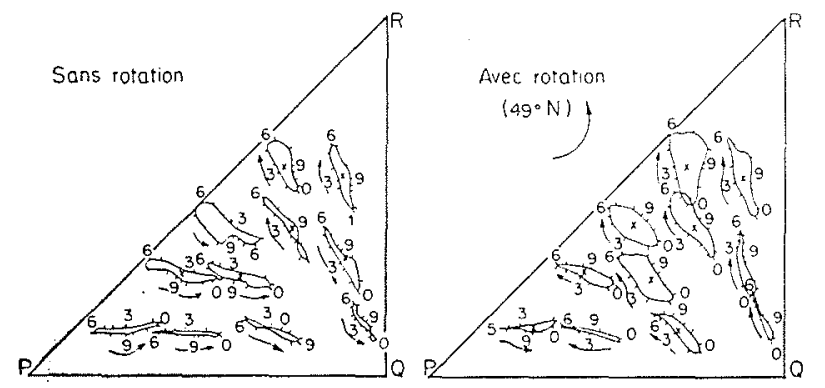

FIG. 5

Période de la marée : $14,15 \mathrm{~s}$.

Amplitude du batteur : $120 \mathrm{~mm}$.

Période de la rotation de la plateforme: $36,4 \mathrm{~s}$.

Longucur du côté de l'angle droit : $150 \mathrm{~km}$.

Profondeur maximum : $110 \mathrm{~m}$.

Essais avec rugosité.
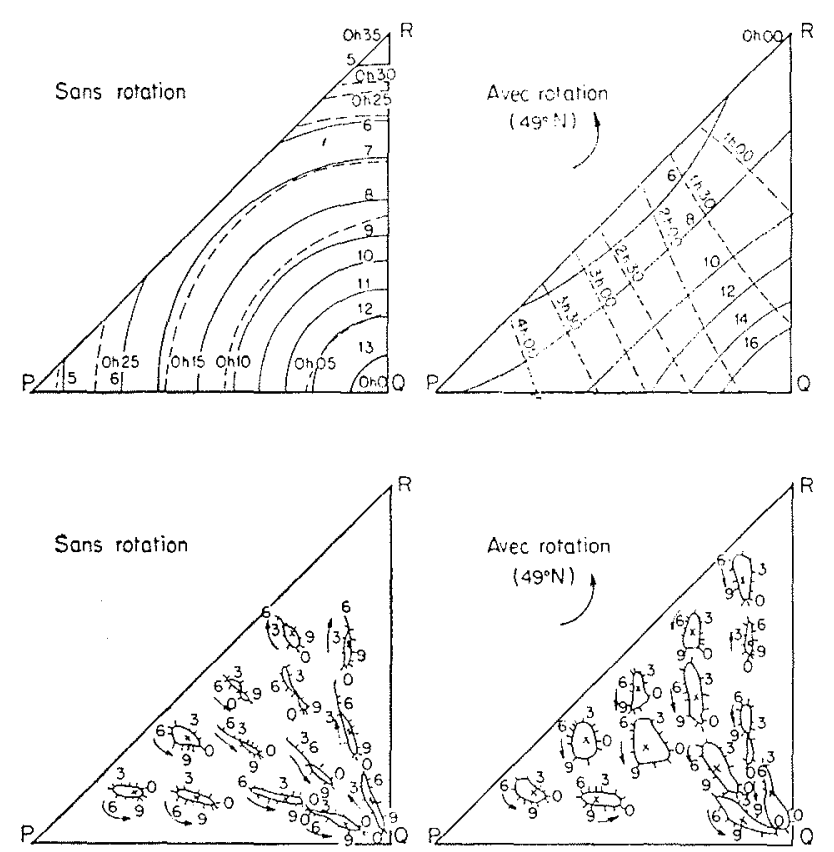

Fig. 7

Période de la marée : 5,77 s.

Amplitude du batieur : $45 \mathrm{~mm}$.

Période de la rotation de la plateforme: $14,8 \mathrm{~s}$

Longueur du côté de l'angle droit : $900 \mathrm{~km}$.

Profondeur maximum : $660 \mathrm{~m}$.

Lssais sans rugosité.

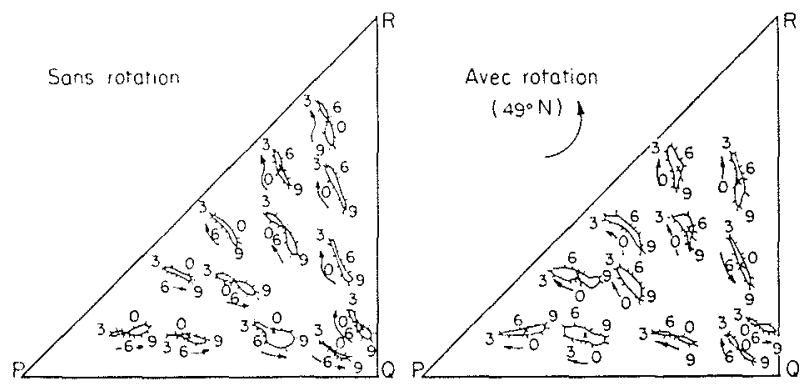

Fic. 6

Période de la marée : $20 \mathrm{~s}$.

Amplitude du batteur : $150 \mathrm{~mm}$.

Période de la rotation de la plateforme: $51,2 \mathrm{~s}$.

Longueur du côté de l'angle droit : $75 \mathrm{~km}$.

Profondeur maximum : $55 \mathrm{~m}$.

Essais cuec rugosité.
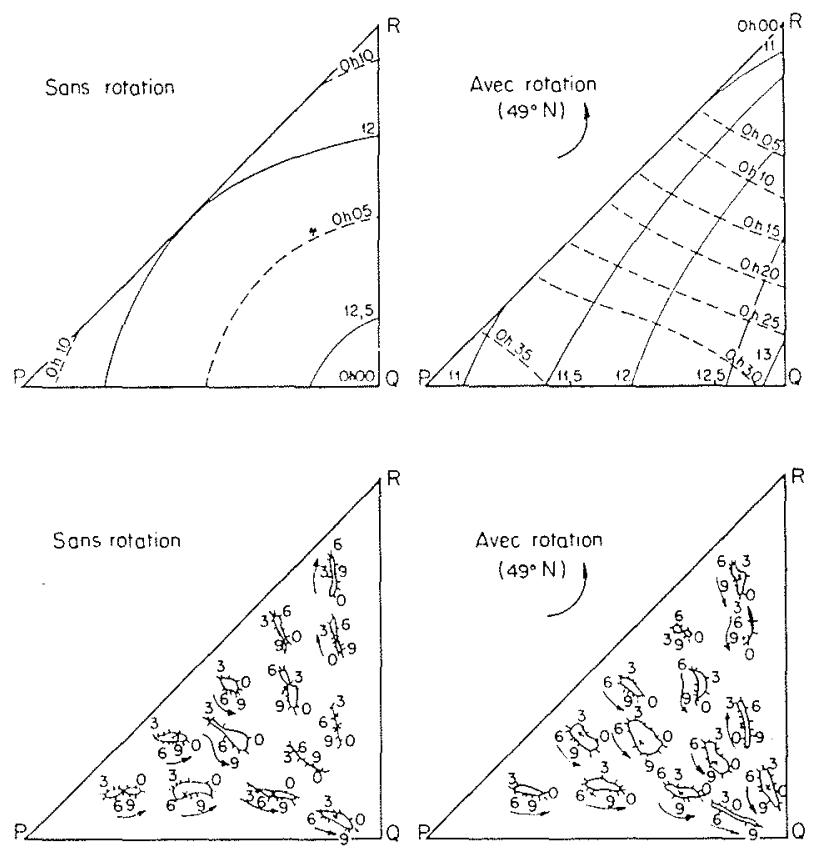

Fig. 8

Période de la marce: $10 \mathrm{~s}$,

Amplitude du balteur : $80 \mathrm{~mm}$.

Période de la rotation de la plateforme : $25,6 \mathrm{~s}$.

Longueur de l'angle droit : $300 \mathrm{~km}$.

profondeur maximum : $220 \mathrm{~m}$

Essais sans rugosite. 

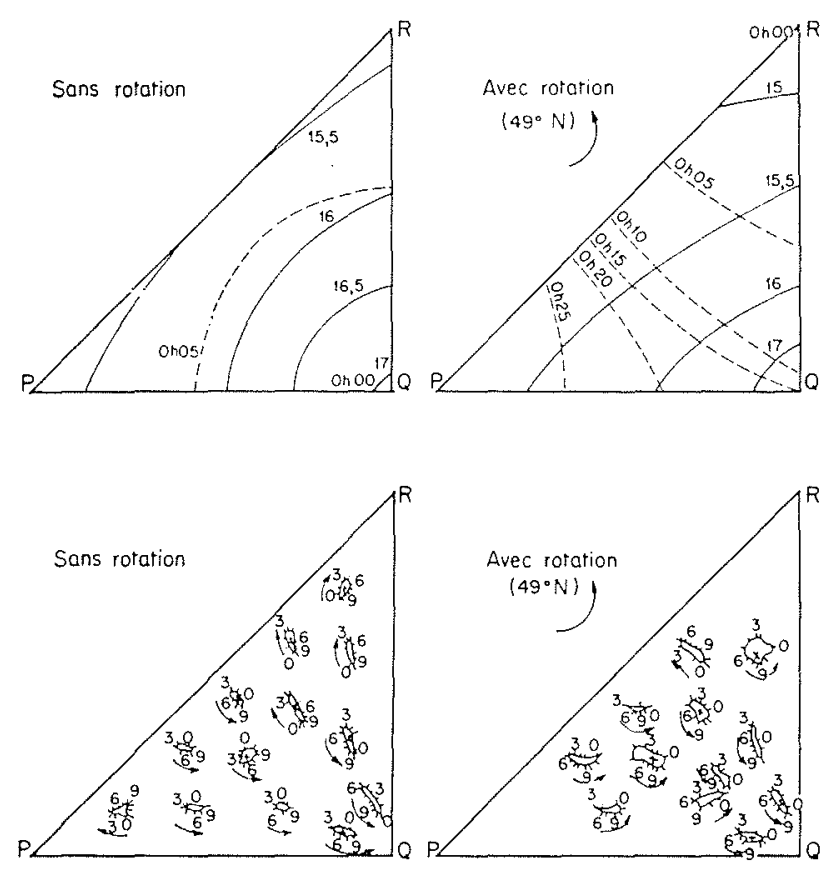

les lignes cotidales sont symétriques par rapport à la bissectrice de l'angle droit. La marée a même amplitude sur les deux côtés de l'angle droit et elle s'y propage avec la même vitesse. Cette obscrvation est valable quelle que soit la valeur du frottement.

Au contraire, le bassin étant animé d'un mouvement de rotation, et le frottement étant important, les lignes d'égal marnage, sous l'action de la force de Coriolis, s'inclinent et forment un angle plus aigu avec le côté $\mathrm{PQ}$ du triangle; il en résulte que la marée est plus importante sur ce côté. Les lignes cotidales s'inclinent au contraire dans l'autre sens; la marée se propage plus rapidement le long du côté RQ que le long du côté PQ. Lorsque les périodes de la marée et de la rotation augmentent, l'effet de la force de Coriolis est de moins en moins sensible. Les deux réseaux de courbes de lignes d'égale amplitude et cotidales sont de moins en moins différents entre les essais avec rotation et sans rotation de la plateforme. L'effet relatif de la force de Coriolis diminue avec la surface de la zone représentée.

Si le frottement est nul, l'effet de la forme de Coriolis sur la différence du marnage entre les deux côtés de l'angle droit et le sommet de celuici est assez faible; le rapport des amplitudes entre les deux sommets $P$ et $R$ du triangle est dans tous les cas voisin de l'unité; ce résultat est très différent de celui obtenu avec frottement. Rappelons que le résultat selon lequel la disparité des marnages des deux côtés d'un bassin tournant est due à l'action conjointe du frottement et de la force de Coriolis, a déjà été observé lors de la marée en canal schématisant Ja Manche.

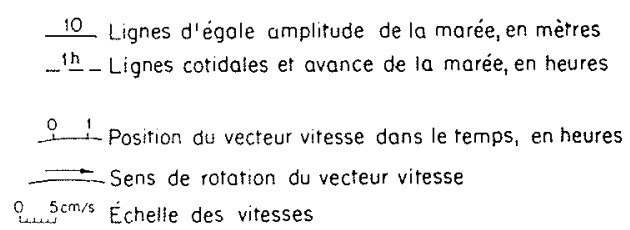

FiG. 9

Période de la marée: $14,15 \mathrm{~s}$. Amplitude du batteur : $120 \mathrm{~mm}$. Période de la rotation de la plateforme : $36,4 \mathrm{~s}$. Longueur du coté de l'angle droit : $150 \mathrm{~km}$. profondeur maximum : $110 \mathrm{~m}$.

Essais sans rugosité.

\section{2。 Etude du marnage relatif.}

\section{a) Frottement important :}

La figure 10 illustre le renforcement de la marée sur le côté $P Q$ sous l'effet de la force de Coriolis pour un frottement important. On a représenté la variation des rapports $\zeta_{\mathrm{P}} / \zeta_{\Omega}$ et $\zeta_{\mathrm{R}} / \zeta_{\mathrm{Q}}$ entre les marnages aux extrémités de l'hypoténuse $P R$ du triangle et le marnage au sommet $Q$ du triangle, en fonction de la longueur correspondant au côté de l'angle droit PQ ou RQ du bassin, dans les trois cas: sans rotation de la plateforme, rotation correspondant à la latitude $49^{\circ} \mathrm{N}$, et vitesse de rotation critique.

Lorsque le bassin tourne, le rapport $\zeta_{\mathrm{P}} / \zeta_{\mathrm{Q}}$ est nettement plus grand (de l'ordre de $100 \%$ ) que celui observé sur le bassin immobile et ceci tant que la longueur du côté du hassin est supérieure à $300 \mathrm{~km}$. Pour les dimensions inférieures, l'écart entre les rapports $\zeta_{\mathrm{P}} / \zeta_{0}$, relatifs au cas avec ou sans rolation, diminue et devient de l'ordre de $5 \%$. Pour les grandes dimensions du bassin et pour une longueur donnée du côté du bassin, le rapport $\zeta_{\mathrm{P}} / \zeta_{\Omega}$ croît avec la vitesse de rotation du bassin; cependant, l'écart relatif entre les essais correspondant à $49^{\circ} \mathrm{N}$ et les essais correspondant aux latitudes critiques est plus faible que l'écart par rapport au cas du bassin immobile, lequel correspond à un océan centré à l'équateur.

L'étude du rapport $\zeta_{\mathrm{R}} / \zeta_{0}$ ne fait pas apparaître de différences systématiques entre les cas avec ou sans rotation. L'influence de la force de Coriolis est done nulle sur les marnages relatifs du côté RQ. Ce résultat intéressant montre que, sur un modèle non tournant représentant le golfe de 

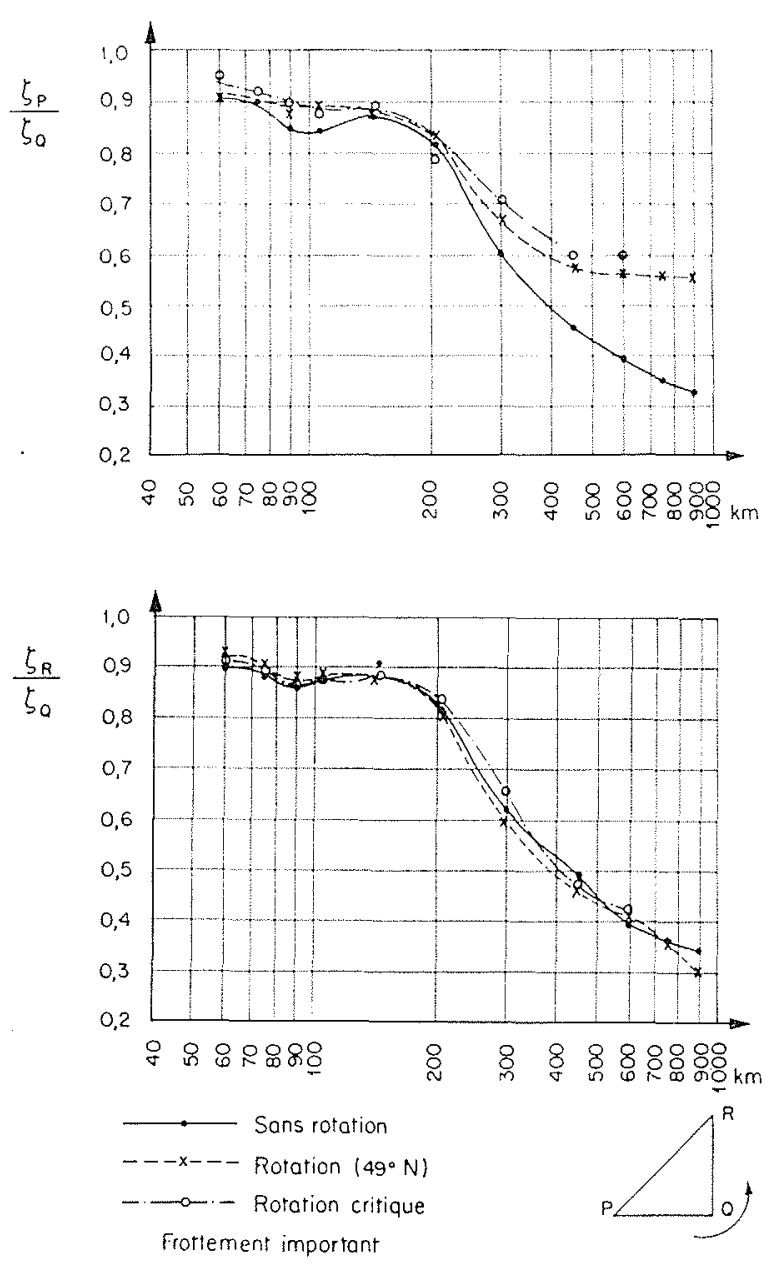

Fig. 10

Variation des rapports des marnages en differents points du triangle en fonction de la longueur: du côté de l'angle droit et de la période de rotation du bassin.

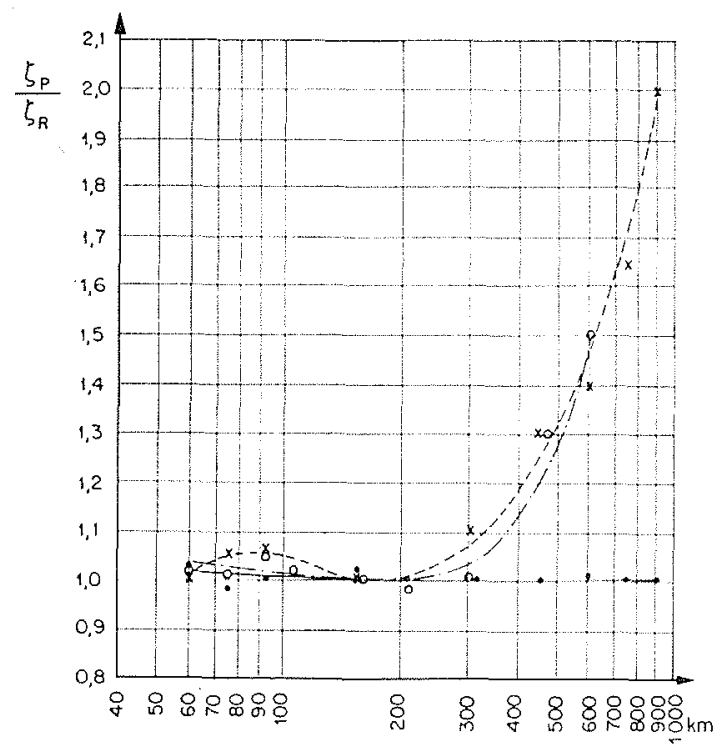

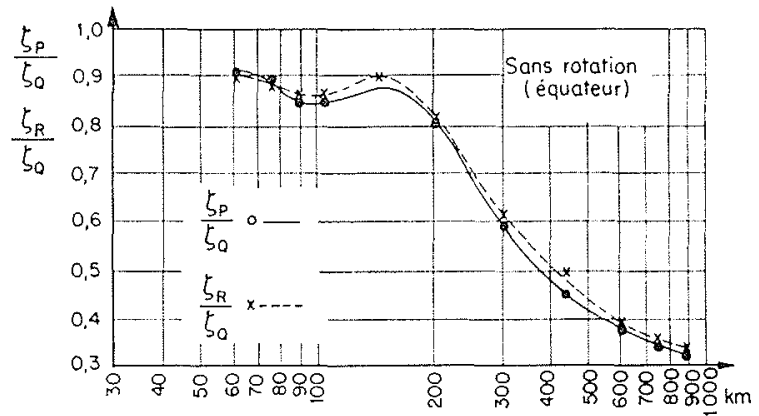
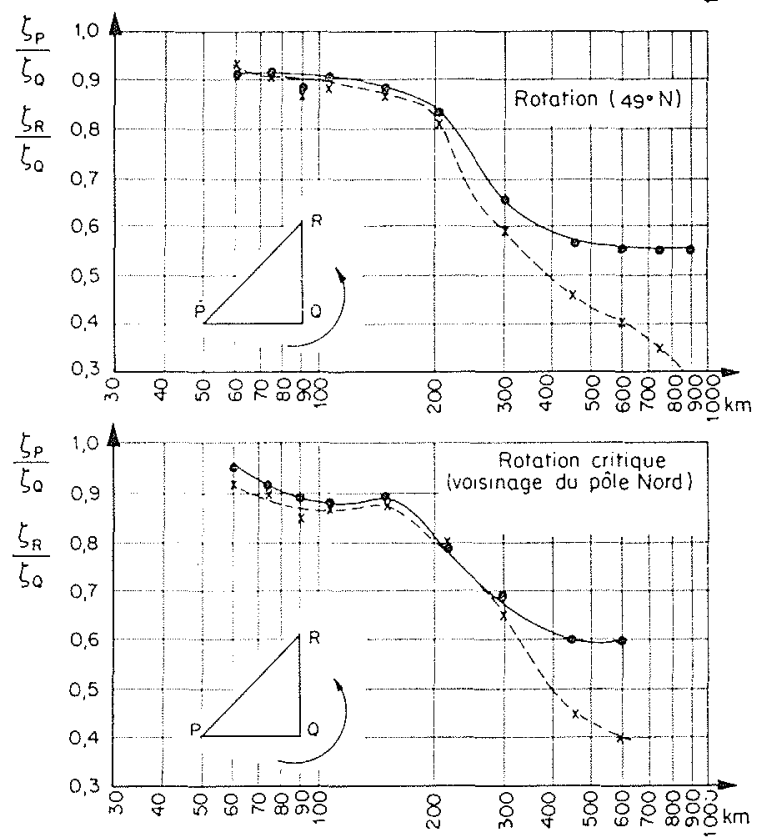

FIG. 12

Variation des rapports des marnages aux différents sommets du triangle, en fonction de la longueur du coté de l'angle droit dans le cas d'un frottement important.

Saint-Malo, on peut reproduire correctement le marnage le long de la côte ouest de la presqu'île du Cotentin, ceci dans l'hypothèse où le frottement est important. La croissance générale des rapports $\zeta_{\mathrm{P}} / \zeta_{\mathrm{O}}$ et $\zeta_{\mathrm{R}} / \zeta_{\mathrm{O}}$ alors que la dimension du bassin diminue, traduit l'effet d'onde stationnaire de la marée dans le bassin.

Pour illustrer l'influence de la force de Coriolis sur le marnage, nous avons tracé sur la figure 11 la variation du rapport $\zeta_{\mathrm{p}} / \zeta_{\mathrm{R}}$ entre les marnages mesurés de part et d'autre de l'extrémité de l'hypoténuse du triangle, c'est-à-dire à chaque extrémité de la pelle du générateur de ma-

\section{Fig. 11}

Variation du rapport $5 \mathrm{p} / \zeta_{1}$ des marnages aux deux sommets de l'hypoténuse en fonction de la longueur du coté de l'angle droit dans le cas d'un frottement important. 


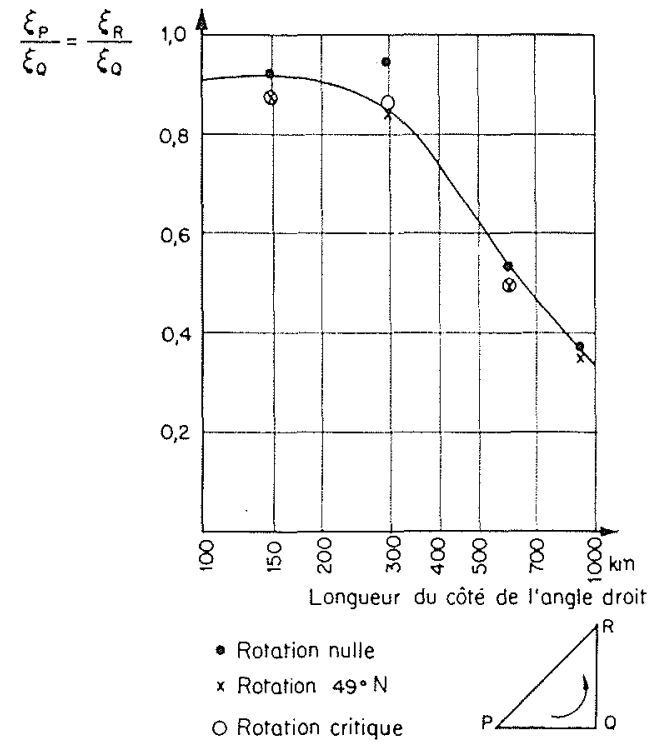

Fig. 13

Rapport des marnages entre les deux extrémités du côté de l'angle droit, en fonction de sa longueur dans le cas d'un frottement nul.

rée. On montre ainsi que $\zeta_{\mathrm{P}}$ et $\zeta_{\mathrm{R}}$ sont peu difrérents dans le cas d'un bassin non tournant, l'onde-marée étant pratiquement symétrique par rapport à la bissectrice de l'angle droit du bassin. Par contre, dans un bassin tournant, le rapport $\zeta_{\mathrm{P}} / \zeta_{\mathrm{R}}$ varie de 2 à 1 lorsque la longueur du côté de l'angle droit décroît de $900 \mathrm{~km}$ à zéro. Pratiquement, le rapport $\zeta_{1} / \zeta_{R}$ est important et supérieur à 1,1 jusqu'à $300 \mathrm{~km}$. Dans les domaines maritimes de moins de $300 \mathrm{Jsm}$ et en particulier pour les bassins de l'ordre de grandeur du golfe de SaintMalo (75 km de longueur environ), le marnage au point $\mathrm{P}$ est d'environ $5 \%$ supérieur à celui au point $R$. On peut en déduire que l'influence relative de la force de Coriolis est de $5 \%$ sur les marnages d'une enceinte de dimension comprise entre 50 et $100 \mathrm{~km}$ et où Je frottement est important.

Enfin, la figure 12 montre le même phénomène en groupant les courbes de variation $\zeta_{\mathrm{P}} / \zeta_{\mathrm{O}}$ et $\zeta_{\mathrm{N}} / \zeta_{\mathrm{g}}$ par égale valeur de la vitesse de rotation de la plateforme. La diminution de l'effet de la force de Coriolis sur les marnages apparaît nettement au-dessous de $200 \mathrm{~km}$.

\section{b) Frottement NUL :}

La figure 13 représente la loi de variation des rapports des marnages aux sommets $P, Q^{\prime}$ et $R$, en fonction de la longueur du côté du bassin. Les marnages $\zeta_{\mathrm{r}}$ et $\zeta_{\Omega}$ sont toujours pratiquement égaux et sont représentés par le même point pour chaque vitesse de rotation de la plateforme. Par comparaison avec les figures précédentes, on remarque que les rapports $\zeta_{\mathrm{P}} / \zeta_{\mathrm{Q}}$ ou $\zeta_{\mathrm{R}} / \zeta_{\mathrm{Q}}$ restent encore voisins de 0,9 pour un bassin de $300 \mathrm{~km}$, alors que, pour cette longueur, ces rapports sont de l'ordre de $0,6-0,7$ lorsque le frottement est important.

\section{c) Conclusion SUR h'ÉTUde DU MaRnage RELATHF :}

Les résultats précédents montrent essentiellement que la disparité des marnages des deux côtés d'un bassin tournant est due à l'action conjointe du frottement et de la force de Coriolis. Ce résultat a déjà été observé Ior's de l'étude de la marée en canal schématisant la Manche.

\section{$3^{\circ}$ Lignes cotidales.}

Les lignes cotidales placées sur les figures 3 à 9 représentent le lieu des passages de la marée par le niveau moyen à un moment donné. Cette définition des lignes cotidales permet une détermination précise du temps de propagation de l'onde-marée, le point de passage de la marée par le niveau moyen étant bien défini sur les enregistrements graphiques. En effet, par suite de la réduction des profondeurs, la pleine mer se propage plus vite que la basse mer; la courbe de marée se déforme en déplaçant son sommet dans le sens de la propagation; il en résulte que des lignes cotidales représentant le lieu des pleines mers sont mal adaptées à la présentation de la propagation de l'onde.

\section{a) Froterement important :}

Quand la plateforme ne tourne pas, l'ondemarée se propage sous forme d'une onde cylindrique dont la ligne de crête est parallèle à l'hypoténuse du bassin triangulaire. Il en résulte que l'onde met pratiquement le même temps pour parcourir les côtés $P Q$ et $R Q$. Lorsque la plateforme tourne et que le frottement de fond est important, dans le cas des bassins de grande dimension de 900 à $600 \mathrm{~km}$ de côté, les lignes cotidales convergent vers un point amphidromique virtuel situé du côté du sommet $R$ du triangle PQR. Ce fait est caractéristique de l'effet de la force de Coriolis sur la propagation de la marée. La zone atteinte la dernière par la marée (ligne cotidale $0 \mathrm{~h} 00$ ) est confondue avec une partie du côté $R Q$ de l'angle droit du triangle.

\section{b) Fro'tTement Nul :}

Le bassin étant immobile, le réseau des lignes cotidales est symétrique par rapport à la bissec- 
trice de l'angle droit comme pour les essais avec frottement. Par contre, la marée présente le caractère d'une onde stationnaire ayant un nœud d'oseillation situé au-delà de l'hypoténuse du triangle. En effet, les temps de propapation de l'onde depuis le générateur de marée jusqu'au sommet de l'angle droit du triangle sont tris courts. Le tableau suivant compare les différences de temps de propagation entre les mêmes essais avec ou sans rugosité de fond.

\begin{tabular}{|c|c|c|c|}
\hline \multirow{2}{*}{$\begin{array}{c}\text { Période } \\
\text { de la } \\
\text { marée inodèle } \\
\text { (s) }\end{array}$} & \multirow{2}{*}{$\begin{array}{l}\text { Longueur } \\
\text { représentée } \\
\text { par le cóté } \\
\text { du bassin } \\
\text { (lkm) }\end{array}$} & \multicolumn{2}{|c|}{$\begin{array}{l}\text { Temps mis par l'onde pour } \\
\text { parcourir le trajet: hypoté- } \\
\text { nuse-sommet de l'angle droit } \\
\text { (en temps nature) }\end{array}$} \\
\hline & & avec rugosité & sans rugosité \\
\hline 5,77 & 900 & 1 h 15 & 0 l1 30 \\
\hline 7,07 & 600 & $1 \mathrm{~h} 00$ & $0 \ln 10$ \\
\hline 10,00 & 300 & $0 \mathrm{~h} 45$ & 0 \ 10 \\
\hline 14,15 & 150 & 0 h 20 & () h 05 \\
\hline
\end{tabular}

Lorsque le bassin tourne, le phénomène est différent. On observe, quelle que soit la période de la marée, une onde qui se propage à partir de l'extrémité $\mathrm{P}$ de l'hypoténuse en se dirigeant ver's l'autre. Les lignes cotidales montrent clairement l'existence d'un point amphidromique virtuel situé au-delà de l'hypoténuse du bassin. Arec rugosité de fond, ce phénomène n'a été observé que pour les bassins de grande surface.

\section{c) TEMPS DE PROPAGATION DE L'ONDE-MARÉE:}

Sur la figure 14, nous avons représenté la variation du temps mis par l'onde-marée pour parcourir les còtés $P Q$ et $R Q$ du bassin, en fonction de la longueur de ces côtés, lorsque le frottement est important. On constate, en ce qui concerne le côté $P Q$, que le temps de propagation ' $\mathrm{T}_{\mathrm{P} Q}$ est fonction croissante de la longueur du côté et ceci d'autant plus que la vitesse de rotation de la plateforme croît. C'est ainsi que le côté de $900 \mathrm{~km}$ de long est parcouru en $1 \mathrm{~h} 15$ environ lorsque le bassin est immobile, et en 2 h 30 lorsque le bassin tourne en correspondant au $49^{\circ}$ de latitude nord. La figure 15 donne la valeur de l'augmentation du temps de propagation en fonction de la longueur de PQ entre les cas avec ou sans rotation. Alors que la différence du temps de propagation est de 1 h 15 pour $900 \mathrm{~km}$, elle est réduite à $5 \mathrm{mn}$ pour $\mathrm{PQ}$ représentant $60 \mathrm{~km}$.

La variation du temps de la propagation $T_{\mathrm{no}}$

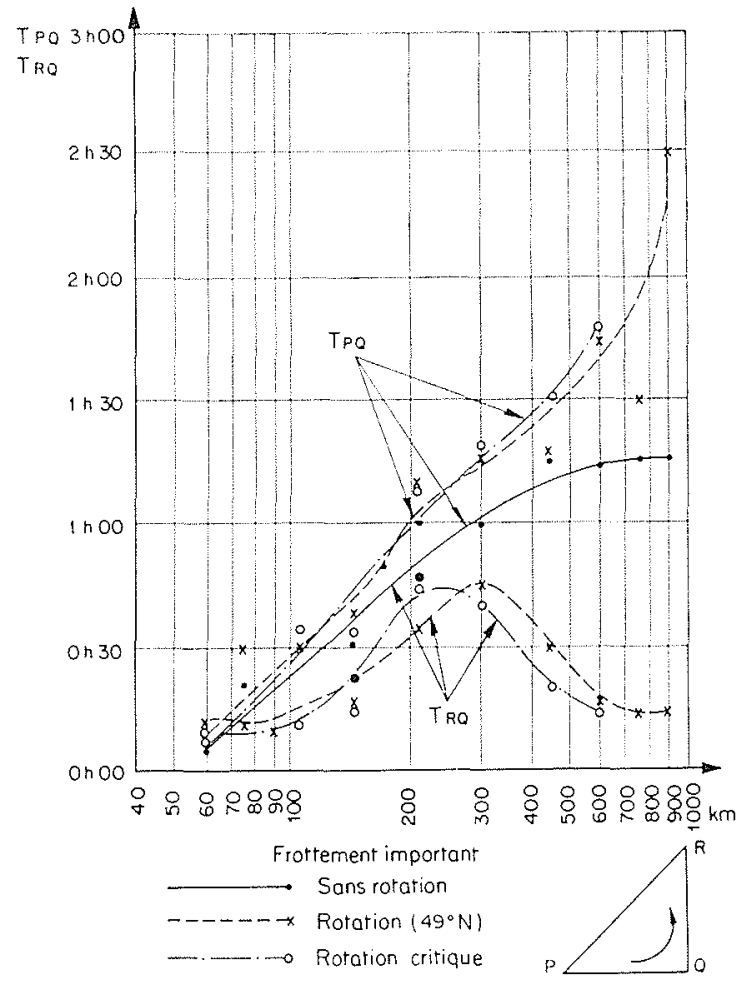

Fig. 14

T'emps mis par l'onde-marée pour parcourir les cotés $P($ et $R Q$ en fonction de la longueur du coté de l'angle droit.

le long du coté $R Q$ est plus complexe, du fait de la faible inclinaison des lignes cotidales par rapport à ce côté (fig. 14). Le bassin étant immobile, les temps de propagation le long de $\mathrm{PQ}$ et $\mathrm{RQ}$, ' $\mathrm{I}_{\mathrm{P} O}$ et $\mathrm{T}_{\mathrm{RO}}$ sont pratiquement égaux. Lor'sque le bassin tourne, le temps $\mathrm{T}_{\mathrm{ro}}$ est faible, car, pour les bassins de grande dimension, une ligne cotidale se confond presque avec le côté RQ, l'onde-marée ayant tendance à se propager parallèlement à la direction du côté $P Q$. Lorsque la longueur du côté $R Q$ diminue et que corrélativement l'influence de la force de Coriolis décroit, le temps de propagation $\mathrm{T}_{\mathrm{R} Q}$ le long de $\mathrm{RQ}$ tend à se rapprocher du temps propagation $\mathrm{T}_{\mathrm{P} O}$ le long de $\mathrm{PQ}$. Il en résulte que la variation de ' $\mathrm{T}_{\mathrm{R} Q}$ présente un maximum de l'ordre de 0 h 45 pour' $R Q$ représentant $250 \mathrm{~km}$ environ. La différence des durées $\mathrm{T}_{\mathrm{ro}}$ avec ou sans rotation du bassin est représentée graphiquement sur la figure 15 .

Lorsque le bassin tourne, $T_{\mathrm{no}}$ est inférieur et 'Tro est supérieur aux durées correspondantes relatives au cas où le bassin est immobile. Il en résulte que l'effet maximum de la force de Coriolis est illustré par l'étude de la différence des temps de passage de l'onde-marée aux points $P$ et R. Cette différence est pratiquement nulle lorsque le bassin est immobile; la figure 16 montre que la différence est de l'ordre de 2 h 15 pour 


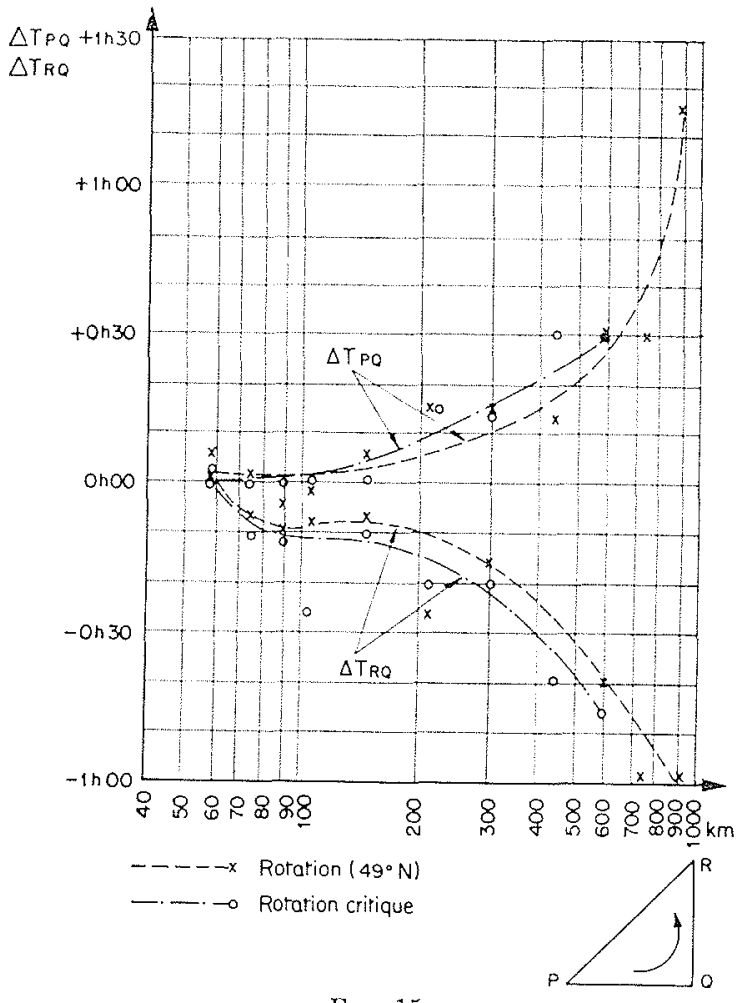

Fig. 15

Augmentation et diminution des temps Tro el Tro mis par l'onde-marée pour parcourir jes cotés $P Q$ et $R Q$, entre les cas avec ou sans rotation avec frottement important.

$900 \mathrm{~km}$; elle tombe à environ $10 \mathrm{mn}$ pour un bassin de l'ordre de $60 \mathrm{~km}$.

Lorsque le frottement est nul, nous savons que le temps de propagation $\mathrm{T}_{\mathrm{P} o}$ est trés faible. L'onde se propage vers le sommet $R$; il n'est pas intéressant d'étudier le temps $\mathrm{T}_{\mathrm{ro}}$. La propagation de l'onde est alors définie par l'étude du temps $T_{\text {Pri }}$, dont la variation est indiquée sur la la figure 16. Pour le bassin de plus de $300 \mathrm{~km}$ de côté, la différence de phase entre les extrémités $\mathrm{P}$ et $\mathrm{R}$ de l'hypoténuse du bassin est d'autant plus grande que le frottement est faible. Ceci est une manifestation de la proximité du point amphidromique virtuel d'autant plus importante que le frottement est faible.

\section{Conclusion sur l'étude de l'amplitude et de la propagation de la marée.}

L'étude de la forme de l'onde-marée caractérisée par l'amplitude maximum $\zeta(x, y)$ en chaque point du domaine, a montré que la figure d'onde obtenue lorsque le bassin ne tourne pas est symétricue par rapport à la bissectrice de l'angle droit du triangle. Le bassin étant animé d'un mouvement de rotation et le frottement étant important, on observe une augmentation du marnage sur un côté de l'angle droit du triangle, tandis que ce marnage reste inchangé sur l'autre coté du bassin. L'onde, qui était cylindrique le long de l'hypoténuse du bassin, devient à profil incliné de la même façon qu'une onde de Kelvin. Cette déformation de l'onde, très sensible $(100 \%)$ pour un bassin de l'ordre de $900 \mathrm{~km}$ de côté, est réduite à $5 \%$ pour un bassin de $60 \mathrm{~km}$ de côté. Au contraire, si le frottement est nul et bien que Ie bassin tourne, l'onde est toujours symétrique par rapport à la bissectrice de l'angle droit.

L'étude des lignes cotidales de la marée définies comme lieu des points de passage de la maréc par son niveau moyen à divers instants, a montré que le réseau de lignes cotidales est symétrique dans le cas d'un bassin immobile. Ce réseau présente un point amphidromique virtuel dans les cas où la plateforme tourne, mais, dans le cas d'un frottement important, cette remarque ne subsiste que pour les bassins de grande dimension (plus de $300 \mathrm{~km}$ de côté). Relativement, l'influence de la force de Coriolis est plus imporlante sur la propagation de la marée que sur son amplitude; le réseau des lignes cotidales est plus modifié que le réseau des lignes d'égale amplitude par la rotation de la plateforme. Le fait principal à retenir est, qu'avec rotation, l'onde met plus longtemps à se propager le long d'un coté de l'angle droit du triangle et moins longtemps le long de l'autre.

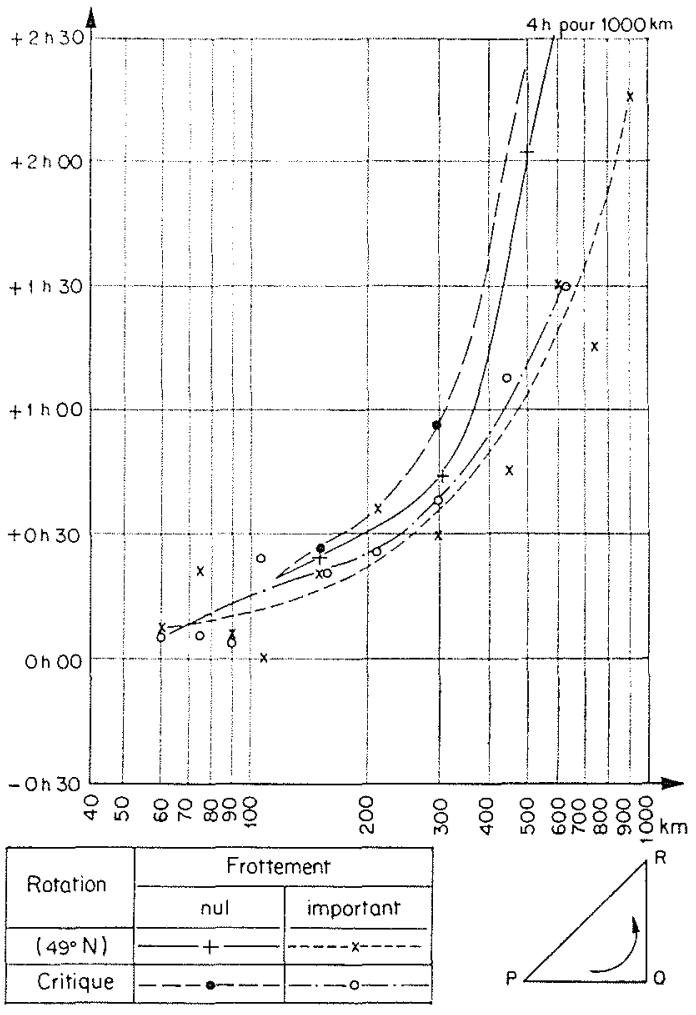

Fici, 16

Difference de phase de l'onde-marée entre les extrémités $P$ et $R$ du bassin. 


\section{VI. - ÉTUDE DES COURANTS}

\section{Généralités.}

A chaque essai, les courants sont relevés en grandeur et direction en 13 points également répartis à la surface du bassin. Le procédé le plus adapté pour représenter facilement le champ de courant est de tracer les "roses do courant », c'est-à-dire l'hodographe du vecteur vitesse au point de mesure et centré sur ce point. Compte tenu de la périodicité du phénomène, l'hodographe se présente sous forme d'une courbe fermée. Pour fixer la position du courant dans le temps, la rose de courant est graduée de 0 à 11 , chaque intervalle représentani une heure-marée, c'est-à-dire $1 / 12$ de la période de la marée, soit $62 \mathrm{mn}$ de temps solaire moyen pour une période de $12 \mathrm{~h} 24 \mathrm{mn}$. Le temps origine correspondant à la graduation zéro a été choisi de façon à coïncider avec l'instant du passage de la pelle du générateur de marée par sa position moyenne au cours de la phase de son mouvement de translation sinusoïdal vers le modèle. Il en résulte que le temps origine correspond à l'heure du maximum de la composante du courant de flot introduit par le générateur de marée normalement à la limite du modèle.

Etant donné la similitude de forme, tant dans l'espace que dans le temps, que présentent les roses de courant d'un essai à l'autre pris dans chacune des trois séries: sans rotation de la plateforme, avec rotation $49^{\circ} \mathrm{N}$, et rotation critique, nous n'avons présenté sur les figures 3 a 9 que les roses de courant obtenues pour quelques essais, pour lesquels la Iongueur de l'angle droit du bassin correspond à 900,300 , 150 et $75 \mathrm{~km}$.

Dans le tracé des lignes cotidales de la marée, nous avions choisi, comme origine du temps, l'heure où la marée passe par le niveau moyen au sommet du triangle, dans le cas du bassin immobile, ou bien l'hetire où la marée finit d'atteindre le côté $R Q$, dans les cas avec rotation et frottement fort, ou atteint le sommet $\mathrm{R}$, dans le cas avec rotation et sans frottement. Pour relier les origines des temps relatives aux lignes cotidales et aux roses de courant, nous avons tracé sur la figure 17 la différence en temps entre ces deux origines horaires, en fonction de la Iongueur représentée par le côté de l'angle droit du bassin. On voit par exemple que, pour le bassin de $900 \mathrm{~km}$ de côté, l'heure zéro des lignes cotidales a lieu $1 \mathrm{~h} 30$ après l'heure zéro des roses de courant, c'est-à-dire 1 h 30 après

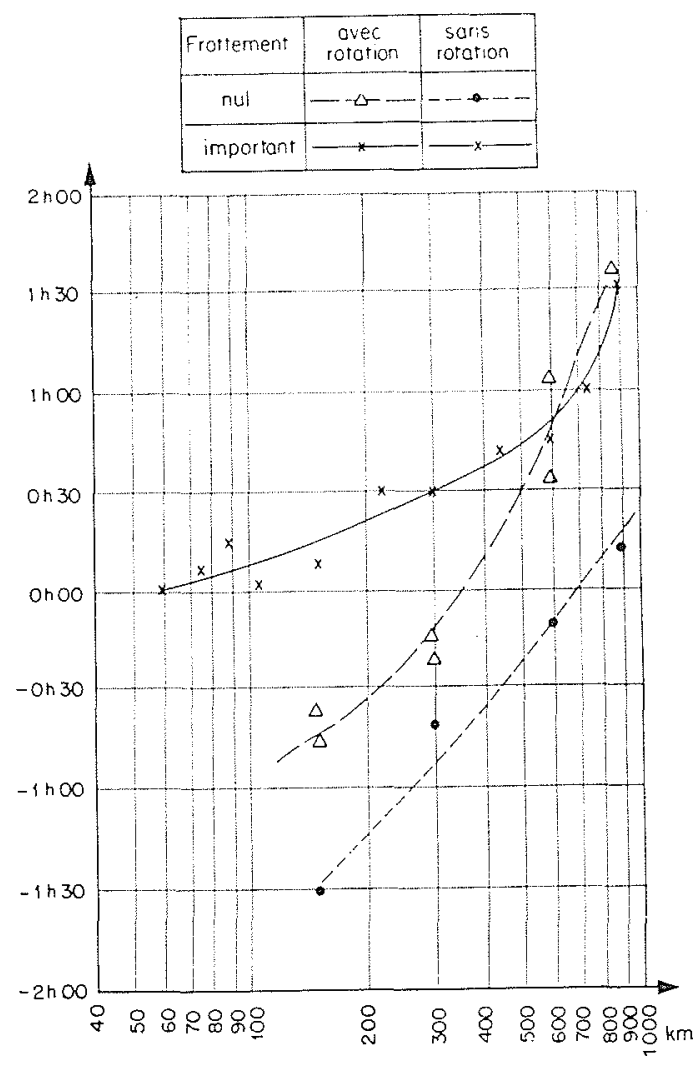

FIG. 17

Retard de l'heure zéro des lignes cotidales sul lheure revo des roses de courant en fonction de la longueur du coté du bassin.

l'heure du maximum de flot de la composante de courant normale au générateur de marée.

\section{Sens de rotation des roses de courant.}

\section{(1) BASSIN IMMOBILE :}

Lorsque la plateforme ne tourne pas, les roses de courant sont grossierement symétriques par rapport à la bissectrice de l'angle droit du triangle. Le sens de rotation du vecteur vitesse est le sens trigonométrique dans la partie située au-dessous de la bissectrice, et le sens opposé de l'autre côté de la bissectrice.

\section{b) Bassin tounnant avec Frottemen't} IMPORTANT:

Les essais montrent que, sur presque la totalité de la surface du bassin, le vecteur vitesse 
tourne toujours dans le sens inverse du sens trigonométrique, c'est-à-dire en sens inverse du sens de rotation de la plateforme. On n'observe que de rares exceptions (fig. 3, 4 et 6) près du sommet $Q$ du triangle; nous verrons qu'elles ne sont pas dues à des erreurs de mesure, mais qu'elles ont un sens physique.

La rotation du bassin avec frottement, donc l'influence de la force de Coriolis ajoutée à celle du frottement, a pour effet d'imprimer au vecleur vitesse une rotation qui s'ajoute à celle déjà observée dans la moitié supérieure du triangle et qui s'oppose dans l'autre moitić à la rotation observée dans le cas où la plateforme est immobile. Il en résulte que, dans la partie du triangle voisine du côté $R Q$, la forme elliptique des roses de courant se modifie dans le sens de la transformation de l'ellipse en cercle: le rapport du petit axe de l'ellipse au grand axe croît. Au contraire, dans la zone voisine du côté $P Q$ du triangle, les ellipses deviennent plus aplaties lorsque la plateforme tourne, puisque la force de Coriolis a pour effet de les faire tourner en sens inverse.

\section{c) BASSIN TOURNANT AVEC FROTTEMENT NUL :}

Lorsque le bassin sans rugosité de fond est animé d'un mouvement de rotation, les roses de courant sont encore toutes parcourues dans le même sens (fig. 7 à 9). Mais ce sens de rotation est le même que celui de la plateforme, c'està-dire qu'il est de sens inverse de celui observé dans les mêmes conditions mais avec frotiement.

Pour les mêmes raisons que précédemment, les roses de courant tendent vers la forme circulaire près des côtés $\mathrm{PQ}$ où le sens du parcours est le même avec ou sans rotation du bassin; au contraire, près du côté RQ, les roses de courant tendent à s'aplatir.

\section{$3^{\circ}$ Conclusion sur l'étude des courants.}

Les résultats précédents montrent que, pour une vitesse donnée de la rotation de la plateforme, les champs de courant sans frottement et avec frottement important sont symétriques par rapport à la bissectrice de l'angle droit du triangle. Nous verrons par la suite que cette remarque qualitative ne s'applique pas au cas des frottements de valeur intermédiaire.

Le fait principal à retenir est que le vecteur vitesse tourne dans le même sens que la plateforme si le frottement est faible, et en sens inverse si le frottement est fort, ceci dans le cas de la présence de la force de Coriolis. Si la force de Coriolis est nulle, c'est-à-dire si le bassin est immobile, quelle que soit la valeur du frottement, le vecteur vitesse tourne dans les deux sens de part et d'autre de l'axe de symétrie du bassin.

Devant l'importance des effets combinés du frottement et de la force de Coriolis, il apparait utile d'étudier le champ de courant pour différentes valeurs du frottement et de la force de Coriolis.

\section{VII. - ÉTUDE DE L'INFLUENCE DU FROTTEMENT}

\section{1 . Généralités.}

Pour illustrer l'influence du frottement, nous présentons sur les figures 18 et 19 les résultats d'essais avec ou sans frottement, dans le cas de la rotation critique pour laquelle la période de la rotation de la plateforme est double de celle de la marée; l'effet de la force de Coriolis est alors exagéré par rapport aux essais précédents. Dans ces essais, l'influence du frottement sur les marnages est assez faible, car les dimensions du bassin sont relativement faibles : 150 et $300 \mathrm{~km}$. Par contre, l'existence du point amphidromique virtuel est nette quand le frottement est nul. La comparaison des champs de courant sur les figures 18 et 19 montre que le sens de rotation du vecteur vitesse s'inverse entre les essais sans et avec frottement, sauf à proximité du sommet $Q$ du bassin.

Lorsque le frottement varie de zéro à une valeur importante, on doit voir le point amphidromique se déplacer de l'axe de symétrie du triangle vers le prolongement du côté Q'R et même disparaître. La modification du champ de courant peut s'effectuer de deux façons :

-.. soit il existe un frottement critique pour lequel les roses de courant sont toutes aplaties et par conséquent correspondent à un changement de sens;

- soit à mesure que le frottement croît, il apparaît une région où le courant tourne en sens inverse de la rotation de la plateforme, cette zone occupant toute la surface du bassin pour un frottement important.

La présence d'une zone très petite où le vecteur vitesse tourne dans le même sens que le bassin quand le frottement est fort, incite à penser que la deuxième solution est valable. Pour le montrer, il suffit d'étudier le champ de cou- 


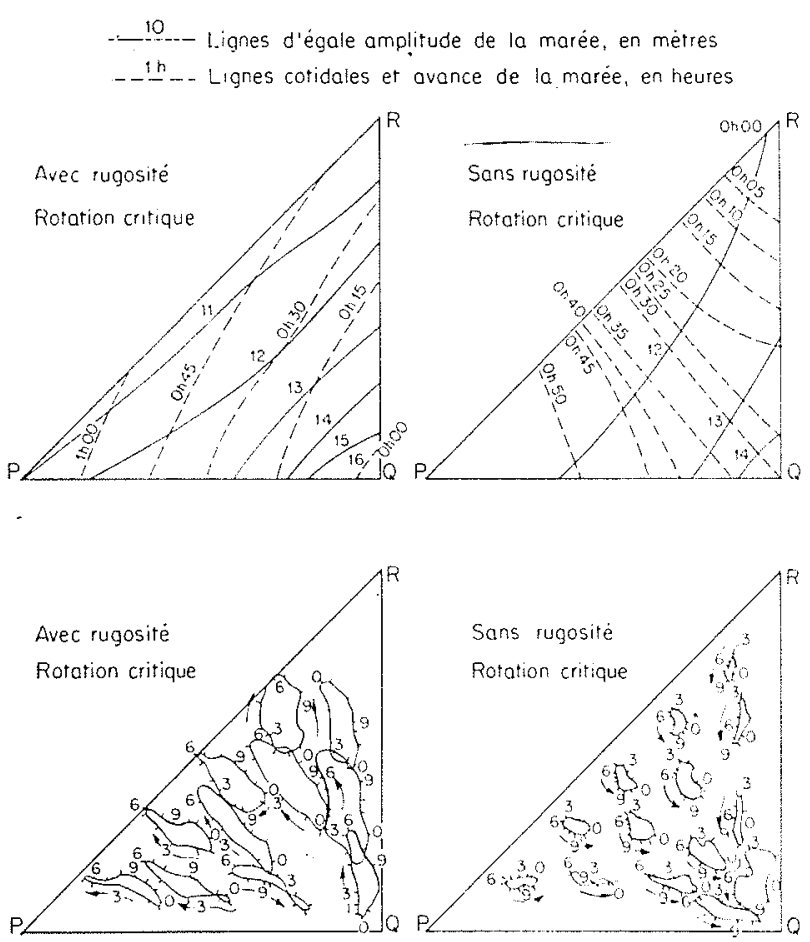

FIG, 18

Période de la marée: $10 \mathrm{~s}$

Amplitude du batteur : $80 \mathrm{~mm}$.

période de la rotation de Ja plateforme: $20 \mathrm{~s}$.

Longueur du côté de l'angle droit : $300 \mathrm{~km}$.

Profondeur maximum : $220 \mathrm{~m}$.

rant pour différentes valeurs de frottement et de la force de Coriolis. Cette étude fait l'objet de la troisième série d'essais annoncée précédemment.

\section{$2^{\circ}$ Essais.}

Les essais complémentaires sont effectues pour quatre périodes de marée 5,77 - 10 - 14,15 - et $18,2 \mathrm{~s}$, et pour le frottement correspondant à des distances entre éléments de rugosité égales à $5 \mathrm{~cm}, 7,5 \mathrm{~cm}, 10 \mathrm{~cm}, 12,5 \mathrm{~cm}, 15 \mathrm{~cm}$ et $17,5 \mathrm{~cm}$. Les résultats de ces essais sont présentés sur les figures 20 à 33 . On peut voir que, pour une distance entre élément de rugosité égale à $5 \mathrm{~cm}$ (frottement le plus fort), la tendance générale des courants est de tourner dans le sens inverse de la rotation du bassin, de même que l'avaient montré les essais avec frottement important. Pour un espace entre élément de rugosité égal à $17,5 \mathrm{~cm}$ (frottement le plus faible), les courants tournent en sens inverse sur presque toute la surface du bassin.

Sur les figures 20 à 23 on a tracé la ligne de séparation entre les deux zones où le vecteur
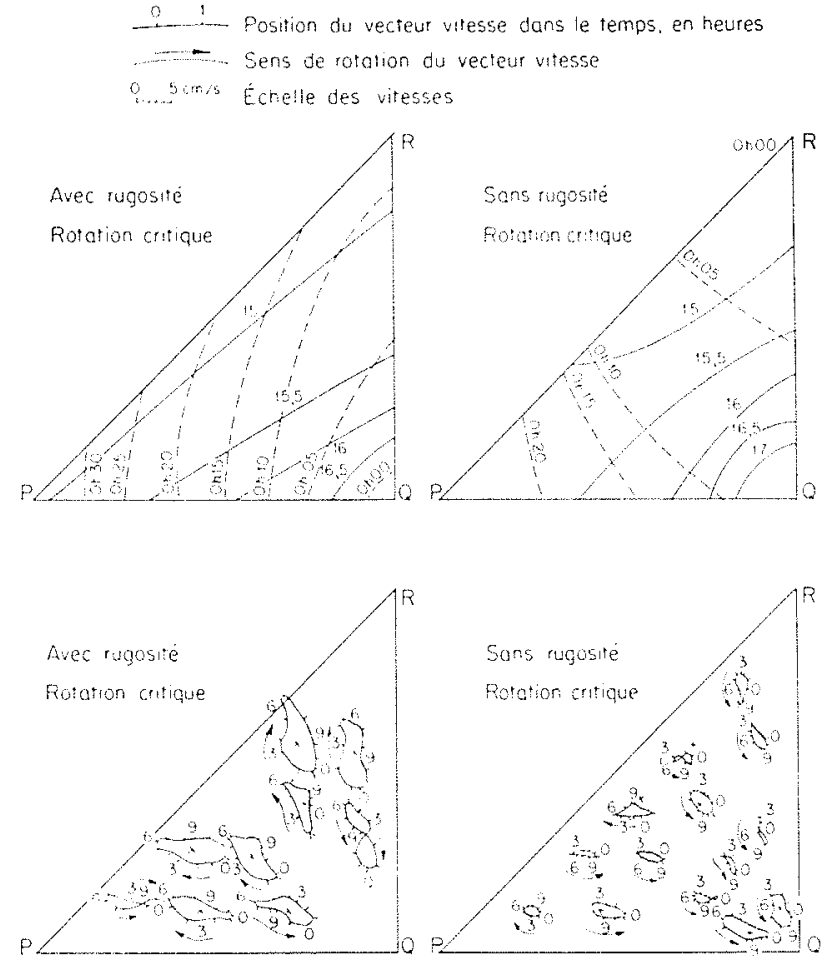

FIIG. 19

Période de la marée : 14,15s.

Amplitude alu batteur : $120 \mathrm{~mm}$.

Période de la rotation de la plateforme : $28,3 \mathrm{~s}$

Longueur du coté de l'angle droit : $150 \mathrm{~km}$.

Profondeur maximum : $110 \mathrm{~m}$.

vitesse tourne en sens différent. La zone où la rotation s'effectue dans le même sens que la plateforme supportani le bassin est très petite pour une rugosité espacée de $5 \mathrm{~cm}$. Elle gagne petit à petit toute l'étendue du bassin lorsque diminue la densité de la rugosité. On voit ainsi apparaitre de façon continue le phénomène de changement de sens de rotation du vecteur vitesse en fonction du frottement en présence de la force de Coriolis. On remarque que lorsque le frottement croît, la zone où les courants tournent en sens inverse du bassin, se manifeste à partir du sommet $R$ du triangle, c'est-à-dire à partir du sommet vers lequel se propage la marée lorsque la force de Coriolis est prépondérante. Corrélativement, la zone où le vecteur. vitesse tourne dans le même sens que le bassin se rétrécit à mesure que croît le frottement, en se réfugiant au sommet $Q$ du triangle, c'estàdire au sommet vers lequel l'onde se dirige lorsque la force de Coriolis est négligeable.

On peut considérer la rotation du vecteur vitesse dans le même sens que le bassin comme une manifestation individuelle de la force de Coriolis. La rotation du courant en sens inverse se présente alors comme un effet du frottement. 
o 1 - Position du vecteur vitesse dans le temps, en heures ${ }_{\text {Omis }}^{5} \mathrm{~cm} / \mathrm{s}$ Échelle des vitesses (fig. 20,21 et 22 )
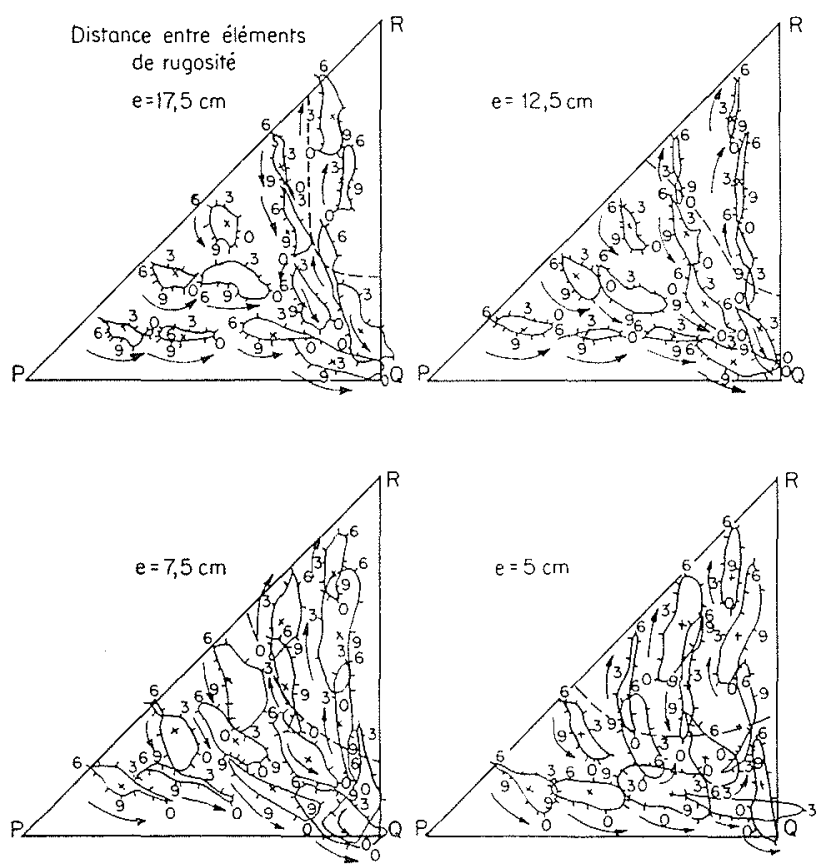

FIG. 20

Période de la marée : $5,77 \mathrm{~s}$.

Amplitude du batteur : $45 \mathrm{~mm}$.

Période de la rotation de la plateforme : 14,8 s.

Longueve du côté de l'angle droit : $900 \mathrm{~km}$.

Profondeur maximum : $660 \mathrm{~m}$.
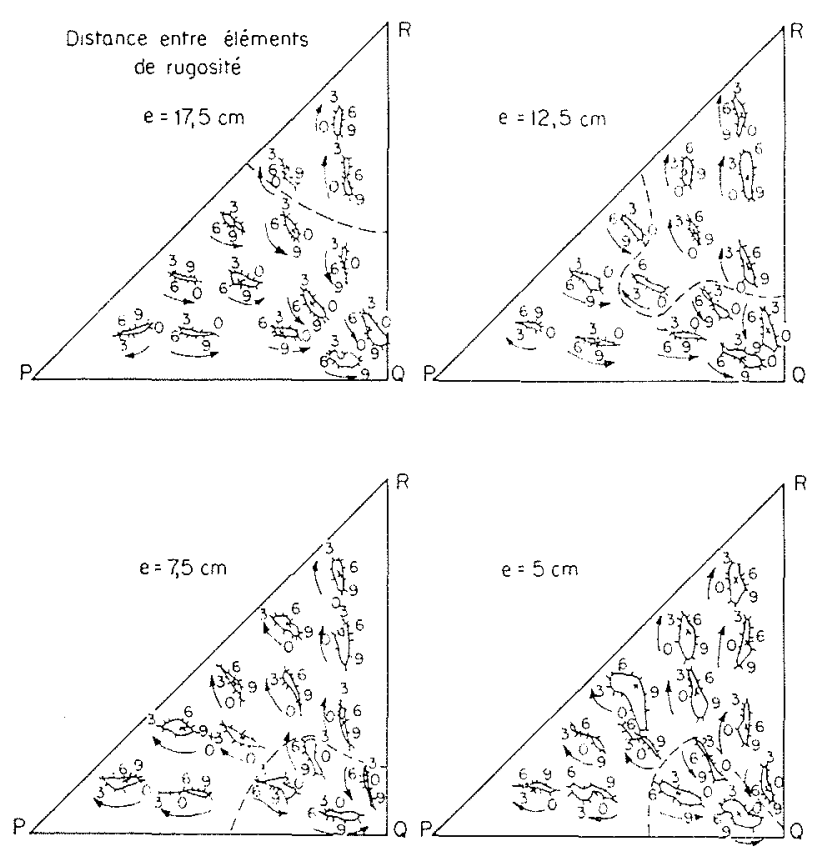

FiG. 22

Période de la marée: $14,15 \mathrm{~s}$

Amplitude du batteur : $120 \mathrm{~mm}$.

Période de la rotation de la plateforme: $36,4 \mathrm{~s}$.

Longueur du côté de l'angle droit : $150 \mathrm{~km}$.

Profondeur maximum : $110 \mathrm{~m}$.
ב- Sens de rotation du vecteur vitesse

_- - L Ligne de changement de sens de parcours des roses de courant
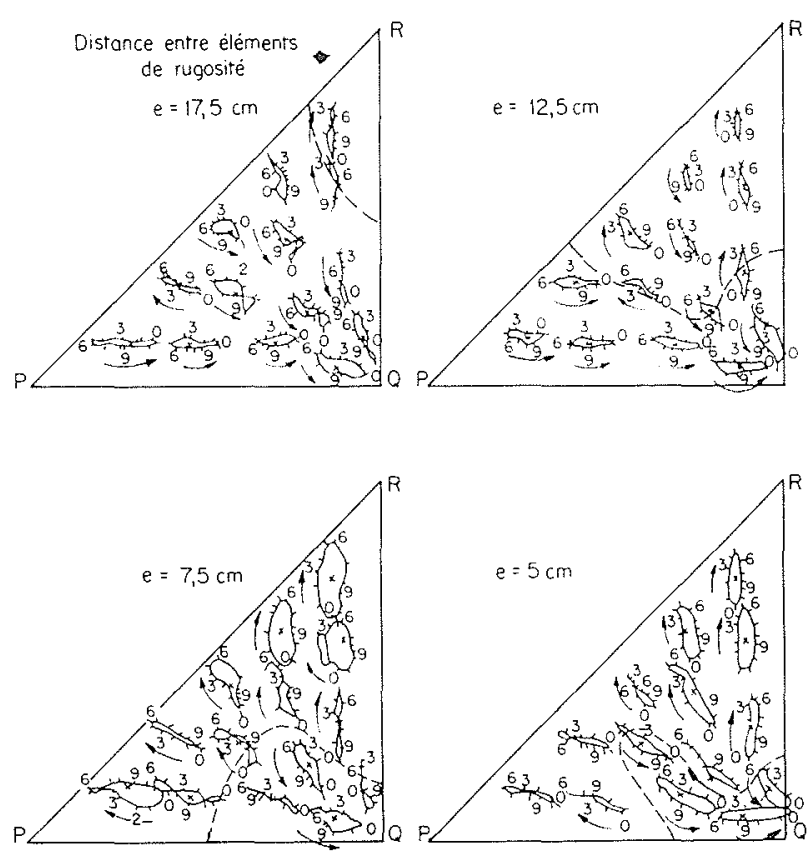

Fig. 21

Période de la maré : $10 \mathrm{~s}$.

Amplitude du batteur : $80 \mathrm{~mm}$

Période de la rotation de la plateforme : 25,6s.

Longueur da colé de l'angle droit: $300 \mathrm{~km}$.

Profondeur maximum : $220 \mathrm{~m}$.
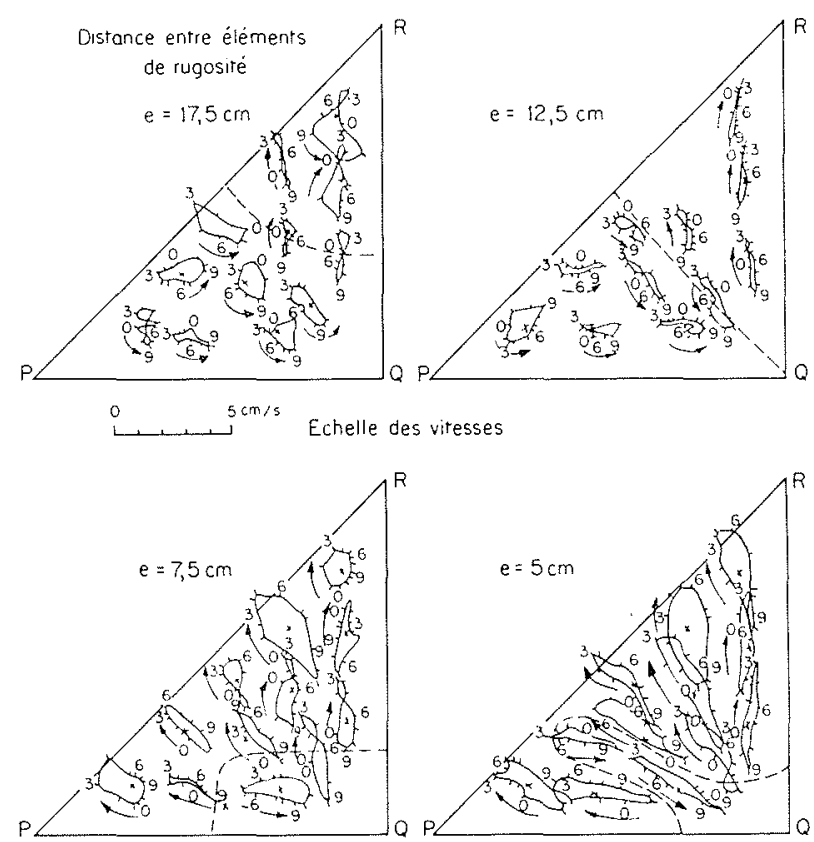

FIC. 23

Période de la marée: $18,2 \mathrm{~s}$.

Amplitude du batteur : $150 \mathrm{~mm}$.

Période de la rotation de la plateforme : $46,8 \mathrm{~s}$.

Longueur du côté de l'angle droit : $90 \mathrm{~km}$.

Profondeur maximum : $66 \mathrm{~m}$. 
De même on a constaté que la propagation de la marée vers le sommel $R$ du bassin est une manifestation de la force de Coriolis senle, la propagation vers le sommet $Q$ étant une conséquence du frottement. Il résulte de ces observations que la zone où se manifeste la tendance «force de Coriolis 》 croît en se propageant en sens inverse de l'onde associée à \& l'effet frottement ». De même, la zone où les courants presentent la tendance « frottement » croit en remontant le parcours de l'onde-marée résultant de la présence de la force de Coriolis. En résumé, la zone à «tendance Coriolis » remonte la marée « avee frottement», la zone à « tendance frottement » remonte la marée «avec Coriolis». On note, en considérant les figures relatives a la densité de rugosité la plus forte, que pour les bassins de grande dimension (900 l:m), le vecteur vitesse ne tourne pas encore dians le même sens sur la totalité du bassin. Dans les premières séries d'essais avec frottement imporlant, on avait effectivement remarqué que le vec-

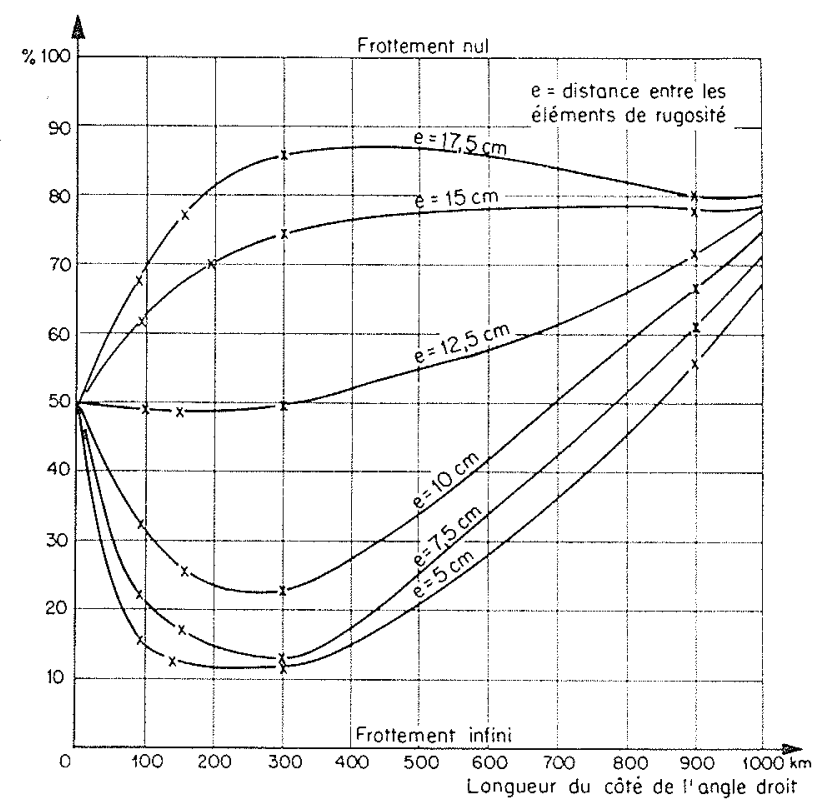

Fig. 24

Pourcentage de la surface du triangle oì le vecteur de vitesse tourne dans le mème sens que le bassin, en fonction de la longueur du côté de l'angle droit et de la rugosite. teur vitesse ne tournait dans le même sens que la plateforme qu’à proximité immédiate du sommet $Q$.

L'influence plus importante de la force de Coriolis se manifeste ainsi en fonction de l'étendue du bassin. Pour chiffrer cette influence, on peut considérer le pourcentage de la surface totale correspondant au sens de rotation semblable à celui de la plateforme, c'est-à-dire intéressée par la force de Coriolis. Si on étudie ce pourcentage en fonction de la longueur du côté du bassin, on obtient une courbe de variation pour chaque valeur de la densité de la rugosité (fig. 24). Lorsque le frottement est nul, la courbe représentative est confondue avec l'ordonnée $100 \%$; Iorsque le frottement est très important, la courbe est confondue avec l'ordonnée $0 \%$ Si la longueur du côté du bassin tend vers zéro, la force de Coriolis devient négligeable, le bassin ne tourne plus et le point figuratif est situé sur l'ordonnée $50 \%$. Pour les valeurs intermédiaires du frottement, et pour les bassins de grande dimension, la force de Coriolis prédomine sur le frottement; le pourcentage de la surface du bassin où le vecteur vitesse tourne dans le même sens que le hassin tend vers la valeur $100 \%$. Au contraire, lorsque la dimension du bassin décroît, les courbes représentatives tendent vers le point thérique $50 \%$. Pour les frottements relativement faibles (distance entre éléments de rugosité égales à 17,5 et $15 \mathrm{~cm}$ ), on observe une décroissance continue de la surface de Ia zone intéresséc par l'effet Coriolis, au fur et à mesure que diminue la longueur du bassin. Pour les frottements relativement forts (distances égales à 5 ou $7,5 \mathrm{~cm}$ ), apròs une décroissance de la zone à «effet Coriolis », on observe une augmentation de cette surface de facon à se rapprocher de la valeur $50 \%$ correspondant au cas sans rotation. Enfin le cas intermédiaire $(e=12,5 \mathrm{~cm}$ ) montre que les deux zones où les vecteurs vitesse tournent en sens opposé ont des surfaces à peu près identiques et ceci pour toute une série de bassins de longueur inférieure à $300 \mathrm{~km}$. Dans ce cas intéressant, le champ de courant sur un bassin fixe est semblable au champ de courant sur un bassin tournant en ce qui concerne le sens de rotation du vecteur vitesse.

\section{VIII. - CONCLUSION}

Les études de marée dans le bassin triangulaire tournant ont pour but la détermination et la précision de l'influence relative de la force de Coriolis par rapport aux autres forces hydrodynamiques en fonction des dimensions linéaides de l'aire océanique considérée. Le bassin 
d'essais est concu de facon à représenter une zone marine de dimensions variables par simple changement de l'échelle des temps, e'està-dire pratiquement par modification de la période de la marée modèle et de la vitesse de rotation de la plateforme supportant le bassin. Les résultats de l'étude sont en toute rigueur relatifs seulement à un bassin déterminé et pour des conditions aux limites donnces, mais peuvent être étendus aux aires marines de formes différentes, pourvu que soit conservé le même type de conditions aux limites au fur et à mesure que l'on modifie l'emprise de la zone marine étudiée.

Pour les aires océaniques de 300 à $1000 \mathrm{~km}$ de longueur, l'influence conjointe de la force de Coriolis et du frottement se traduit par une déformation de ponde-maré, avec aumentation très sensible de 20 à $100 \%$ de l'amplitude de la marée sur les côtes sud du bassin si celui-ci est supposé dans l'hémisphère nord terrestre. Au contraire, sur les côtes est de la zone océanique, la force de Coriolis n'introduit pas de modification en ce qui concerne l'amplitude de la marée. L'onde-marée du type cylindrique sans force de Coriolis prend un profil incliné lors de l'adjonetion de la force de Coriolis. Iorsque le hassin a des dimensions inférieures à $150 \mathrm{~km}$, l'influence de la force de Coriolis sur les marnages ne se traduit plus que par une légère modification de l'ordre de $5 \%$, donc pratiquement négligeable.

Le frottement seul n'a pas d'influence particulière sur le marnage quand la plateforme est immobile. De même, la force de Coriolis seule n'amène aucune disparité du marnage entre les còtés opposés de l'aire marine étudiée.

Le réseau des lignes cotidales de la marée traduisant le phénomène de propagation de l'onde est plus perturbé par la présence de la force de Coriolis que le réseau des lignes d'égales amplitudes de la marée. En effet, si le frottement est important, dans les bassins de l'ordre de 300 à $1000 \mathrm{~km}$ de côté, on voit nettement se dessiner la présence d'un point amphidromique virtuel au nord de la zone océanique. Il en résulte que le temps de propagation de la marée le long des côtes sud du bassin est augmenté sous l'effet de la force de Coriolis. Dans ce cas, les lignes cotidales ayant la direction générale nordsud, la marée aborde simultanément tous les points des côtes est du bassin, au lieu de se propager régulièrement le long de ces dernières, comme dans le cas sans force de Coriolis. Lorsque la dimension du bassin est inférieure à $100 \mathrm{~km}$, l'influence de la rotation terrestre sur la propagation de la marée se traduit encore par une différence de l'ordre de 10 minutes entre les temps de parcours des côtés sud et est du bassin, ces deux temps étant égaux chacun à 20 minutes sans force de Coriolis.
En l'absence du frottement, les points amphidromiques virtuels se forment, quelle que soit la dimension du bassin, lorsque celui-ci tourne, la marée se propage d'une extrémité à l'autre du générateur de marće. Si la force de Coriolis est nulle, sans frottement l'onde est stationnaire.

L'étude des vitesses fait apparaître une modification profonde du champ de courant due à l'influence de la force de Coriolis. Compte tenu de la forme symétrique du bassin par rapport à la bissectrice de l'angle droit du triangle, le champ de courant est symétrique lor'sque le bassin est immobile, quelle que soit la valeur du frottement. L'adjonction de la force de Coriolis au frottement a pour effet :

-- D'une part, de changer le sens de parcours des roses de courant, de facon à le rendre opposé au sens de rotation de la plateforme, el ceci sur presque toute l'étendue du bassin;

- D'autre part, de modifier la forme des roses de courant en corrélation avec le déplacement spatial de la ligne où les roses de courant se réduisent à un segment de droite. Le caractère giratoire des courants est accentué dans les régions où l'effet de la force de Coriolis s'ajoute à l'effet giratoire provenant des autres conditions hydrodynamiques et géométriques.

Ces résultats sont modifiés si le frottement est nul. Dans ce cas, le bassin étant animé d'un mouvement de rotation, le vecteur vitesse tourne partout dans le même sens que le bassin.

On peut conclure que la valeur du frottement influe très profondément sur le champ de courant, d'où la nécessité de l'étudier pour différentes valeurs relatives du frottement et de la force de Coriolis. Cette dernière investigation montre l'apparition d'une ligne de séparation des deux zones dans lesquelles le sens de rotation du vecteur vitesse est inverse. Cette ligne de démarcation se déplace sur la surface du bassin en fonction de l'importance du frottement. La zone où le vecteur vitesse tourne en sens inverse de celui du bassin apparaît au sommet nord du bassin quand le frottement croit; ensuite la zone où le vecteur vitesse tourne dans le même sens que le bassin est reléguée vers le sommet sudest du bassin lorsque le frottement devient très important.

En résumé, cette étude expérimentale montre que, dans le phénomène de marée, on ne doit pas sous-estimer l'influence du frottement. Les résultats théoriques ou de calculs de marée comparatifs, entre les cas avec ou sans force de Coriolis efrectués avec un frottement nul, peuvent donner 
lieu à des interprétations erronées. En résumé, si l'introduction d'un paramètre frottement incorrect a peu d'influence dans le phénomène des marées sans force de Coriolis, l'imprécision sur la valeur du frottement attribuée à un phénomène étudié où la force de Coriolis entre en jeu, peut conduire à des résultats différents de ceux recherchés.

\section{DISCUSSION}

Président : M. Lacombe

M. le Président remercie vivement M. Bonnerlule d'avoir présenté la communication à laquelle il a collabore.

M. Brnel demande si, en dehors des résultats expérimentiux présentes par MM. Valemuois et Bonneflile, on a pu éablir théoriguement l'influence comparée de la force de frottement et de la force de Coriolis et l'influence combince de ces deux forces.

M. Fibavchento pépond à M. Binder que M. Takano poursuit actuellement, au Laboratoire de Mécanique des Fluides de l'Université de Grenoble, une tentative pour mettre en évidence ct calculer les robles respectifs des coefficients de frottement et de la fore de Coriolis. Ce problème, qui intéresse l'ingénicur, est difficile et le chercheur, malgré sa valeur, n'a pas encore obtenu de résultant concluant. M. TAKANo a tenté d'introduire un frottement linéaire proportionnel au vecteur vitesse a chaque instant. Cette recherche est faite en liaison ave le travail de MM. YaLmmors et BONNErrLes, et donnera lieu peut-être, dans un avenir plus ou moins éloigne, a une publication commune importante.
I. ScHolindie confirme que le frottement joue an liès grand rôle et que le manque de frottement, surtout dans les modéles a fond mobile, dans le cas où la force de Coriolis n'est pas tres importante, pent donner des lignes de courant entierement differentes de la nature.

Dans le cas d'une grande étendue sans contrainte, il $\checkmark$ a an effet cumulatil des forces de frottement, qui dome une courbure des lignes de courant autre que dans la realite.

De son côté, M. Boxxerille remarque que l'étude de la mare dans un bassin triangulaire a eu l'avantage de permettre l'utilisation du même modèle avec différentes dimensions. Mais il estime, d'autre part, qu'il serait intéressant d'étudier la marée sur un bassin rectangulaire, qui serail beacoup plus apte au calcul que le cas du triangle.

M. Ie President exprime sa satisfaction de voir tout un ensemble de chercheurs se pencher sur ce problème très difficile, sur lequel on peut espérer avoir bientôt des vues tres précises et des resultats difinitifs.

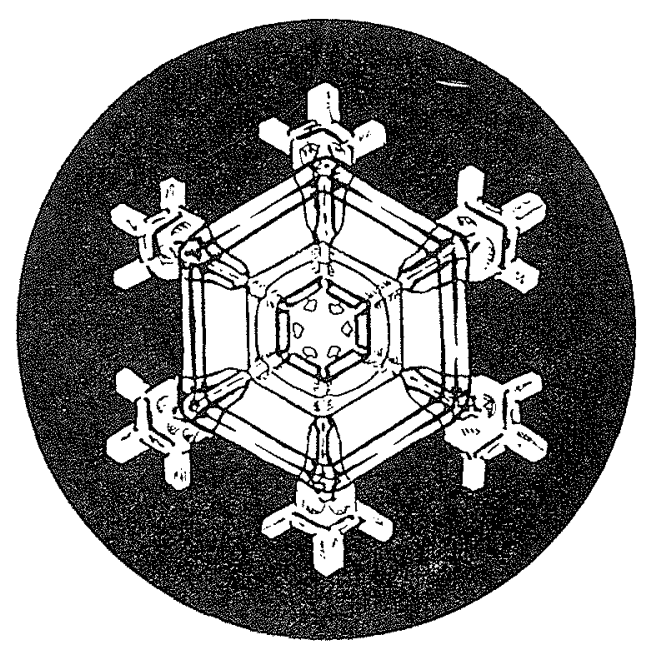

